\title{
Lessons from $T^{\mu}{ }_{\mu}$ on inflation models: Two-loop renormalization of $\eta$ in the scalar QED
}

\author{
Ayuki Kamada $\odot$ and Takumi Kuwahara $\odot$ \\ Center for Theoretical Physics of the Universe, Institute for Basic Science (IBS), Daejeon 34126, Korea
}

(Received 27 August 2020; accepted 28 April 2021; published 1 June 2021)

\begin{abstract}
A nonminimal coupling $\eta$ has been attracting growing interest particularly in the context of inflation models, though its quantum nature is not clear yet. We study the renormalization of $\eta$ in the scalar quantum electrodynamics (QED). There are one-loop diagrams, which could give the finite correction to $\eta$ in the scalar QED. We find that they cancel with each other. We also find no inhomogeneous term of the renormalization group equation (RGE) at the two-loop level, while there could be the two-loop order finite correction. This is similar to other theories, where an inhomogeneous term of the RGE appears only at a higher-loop order: e.g., four-loop order in $\lambda \phi^{4}$ theory.
\end{abstract}

DOI: 10.1103/PhysRevD.103.116001

\section{INTRODUCTION}

Properties of the energy-momentum tensor $T_{\mu \nu}$ are of particular interest in quantum field theory even in the flat spacetime. It determines the linear response of matter to external gravitons. It is a single place where we can study a nonminimal coupling of a scalar field $\phi$ to gravity, $\xi R \phi^{2}(R$ : Ricci scalar), in the flat spacetime. It provides an improvement term, $-\left(\partial_{\mu} \partial_{\nu}-g_{\mu \nu} \partial^{2}\right) \phi^{2}$, to $T_{\mu \nu}$ in the flat spacetime. The improvement term does not change the conservation of $T_{\mu \nu}$ nor the corresponding Poincaré algebra [1,2].

The improvement term, on the other hand, changes the trace of the energy-momentum tensor $T^{\mu}{ }_{\mu}$ by $2 \eta \partial^{2} \phi^{2}$. Here $\xi=\xi_{c}+\eta /(d-1)$ with the conformal coupling being $\xi_{c}=(d-2) /(4(d-1))$ in $d$ dimensions. The improvement term leads to an uncertainty of $T_{\mu \nu}: T_{\mu \nu}$ that couples to gravity does not need to satisfy $T^{\mu}{ }_{\mu}=0$ even if matter is at the conformal fixed point. Meanwhile, the improvement term is subject to renormalization, although $T_{\mu \nu}$ is naïvely expected to be finite due to its conservation. Namely, one has to renormalize $\eta$. There would also be finite corrections to $\eta$ besides the divergences that should be renormalized. Renormalization is twofold throughout this article: the finite correction and renormalization group equation (RGE).

The renormalization of $\eta$ is of interest as it is and studied intensively in $\lambda \phi^{4}$ theory [3-5]. The key is that the RGE of $\eta$ is inhomogeneous. The inhomogeneous term arises from

Published by the American Physical Society under the terms of the Creative Commons Attribution 4.0 International license. Further distribution of this work must maintain attribution to the author(s) and the published article's title, journal citation, and DOI. Funded by SCOAP ${ }^{3}$. the renormalization of trace-anomaly terms (i.e., composite operators [6-9]). The trace anomaly is associated with violation of conformal symmetry at a quantum level and provides additional terms to $T^{\mu}{ }_{\mu}[1-5,10-16]$.

The inhomogeneous term of the RGE induces the inhomogeneous solution of $\Delta \eta$ irrespectively of our initial choice of $\eta$ and thus $\Delta \eta$ is a quantum-induced value of $\eta$ [17]. The inhomogeneous solution would lead to an important consequence in phenomenology. A small change in the nonminimal coupling would lead to a detectable change in the prediction of the inflation dynamics: the chaotic inflation with a simple power-law potential [18], whose large tensor-to-scalar ratio is disfavored by measurements of cosmic microwave background (CMB) anisotropies [19,20], gets viable again just with $\xi \sim$ $-10^{-3}[21-23] .{ }^{1}$ The small nonminimal coupling is more plausible, particularly if it is generated regardless of our choice of the initial value. This motivates us to study the inhomogeneous term of the RGE of $\xi$. The RGE of the nonminimal coupling $\xi$ have been studied in the context of inflation [25-32]. These studies have mainly focused on the homogeneous term of the RGE of $\eta$, which accounts for one part of the inhomogeneous term of the RGE of $\xi$. On the

${ }^{1}$ The minus sign originates from our convention following Refs. [5,24]: the metric signature is $(+,-,-,-)$; the EinsteinHilbert action with a free singlet scalar is

$$
\begin{aligned}
S_{\mathrm{E}-\mathrm{H}}= & -\frac{M_{\mathrm{pl}}^{2}}{2} \int d^{4} x \sqrt{-g} R \\
& +\int d^{4} x \sqrt{-g}\left(\frac{1}{2} g^{\mu \nu} \nabla_{\mu} \phi \nabla_{\nu} \phi+\frac{1}{2} \xi R \phi^{2}\right),
\end{aligned}
$$

with the reduced Planck mass $M_{\mathrm{pl}}$; and the four-dimensional conformal coupling is $\xi_{c}=+1 / 6$. 
other hand, they seem to have missed the inhomogeneous term of the RGE of $\eta$, which accounts for the other part of the inhomogeneous term of the RGE of $\xi$.

We compute the inhomogeneous term in a systematic way by use of flat-spacetime quantum field theory and dimensional regularization. To be more specific, we calculate the Feynman diagrams with an insertion of the trace of the energy-momentum tensor $T^{\mu}{ }_{\mu}$. Dimensional regularization provides an ultraviolet regulator $\epsilon=4-d$ by the analytic continuation of the number of spacetime dimensions $d$. The trace of the $d$-dimensional flat-spacetime energy-momentum tensor could include the terms proportional to $\epsilon$, and then the renormalization of composite operators gives the trace anomaly. This approach enables us to incorporate the corrections from the trace anomaly.

The inhomogeneous term is expected to appear first at a higher-loop order: four-loop order in $\lambda \phi^{4}$ theory [3-5]; and at least two-loop order in the two scalar theory and Yukawa theory [17]. Meanwhile, in the scalar quantum electrodynamics (QED), $T^{\mu}{ }_{\mu}$ contains the gauge kinetic term proportional to $\epsilon$. At first glance, this term would provide a finite correction to $\eta$ even at the one-loop level. However, as shown in this article, there is no one-loop finite correction. Thus, we expect the inhomogeneous term of the RGE of $\eta$ also vanishes at the two-loop order. We explicitly confirm it by computing the two-loop divergent correction to $\eta$. We also note how our results are expected to be generalized to the scalar quantum chromodynamics (QCD). Note that for the fermion QED and QCD, the trace anomaly is known at all orders [12-14,16], but not for the scalar QED or QCD.

Some inflation models prefer a large nonminimal coupling, such as Higgs inflation [33-36]. In these models, it is important to include contributions of the nonminimal coupling to its own RGE. It is not straightforward to apply our flat spacetime approach to investigating such contributions, because we need to incorporate the coupling of matter to Weyl degrees of freedom beyond the linear response. It is also known that the theories with the large nonminimal coupling have a cutoff scale much lower than the Planck scale [37-41], and would not have a perturbative picture above the cutoff scale. This situation would be cured by introducing $R^{2}$ term [42-45]. The RGE of the nonminimal coupling is formulated in the presence of the $R^{2}$ term by the use of an ingenious field redefinition [46], but the corrections from the trace anomaly seem not to be incorporated in their formulation. It is worthwhile to take into account the trace anomaly in their formulation by the use of the Fujikawa method [47-49] or by our approach. The anomaly would appear from the Jacobian of the path-integral measure under the field redefinition, in the Fujikawa method. Meanwhile, in our approach, there could be terms proportional to $\epsilon=4-d$, and then the anomaly would appear from the divergent corrections to the composite operators.
This article is organized as follows. In the next section, we discuss a general property of the RGE of $\eta$ to identify what is to be computed. We show our computation results at the one-loop and two-loop orders in Secs. III and IV, respectively. Section 5 is devoted to discussing the implications of the result. Throughout this article, we adopt the modified minimal subtraction ( $\overline{\mathrm{MS}})$ scheme $[50-52]$ with the spacetime dimensions of $d=4-\epsilon$ and the (modified) renormalization scale $\mu(\tilde{\mu})$.

\section{RGE OF $\eta$}

The scalar-QED action is

$$
\begin{aligned}
S_{\text {mat }}= & \int d^{4} x \sqrt{-g}\left(-\frac{1}{4} g^{\mu \lambda} g^{\nu \kappa} F_{0 \mu \nu} F_{0 \lambda \kappa}+g^{\mu \nu} D_{0 \mu} \phi_{0}^{*} D_{0 \nu} \phi_{0}\right. \\
& \left.+\xi_{0} R\left|\phi_{0}\right|^{2}-m_{0}^{2}\left|\phi_{0}\right|^{2}-\frac{1}{4} \lambda_{0}\left|\phi_{0}\right|^{4}\right)+S_{\text {fix }},
\end{aligned}
$$

with $D_{\mu}$ being the gauge and diffeomorphism covariant derivative. We omit the gauge fixing term $S_{\text {fix }}$ except for the gauge boson propagator as discussed in Ref. [53]. Parameters are a gauge coupling $e_{0}$ (charge being $Q$ ), a scalar mass $m_{0}$, and a quartic coupling $\lambda_{0}$. The subscripts 0 denote the bare fields and couplings. We provide details of multiplicative renormalization in the Appendix A. We remark that in the flat spacetime, the nonminimal coupling $\xi_{0}=\xi_{c}+\eta_{0} /(d-1)$ does not affect multiplicative renormalization.

We define the energy-momentum tensor as a linear response of the matter action to the metric:

$$
T^{\mu \nu}=-\frac{2}{\sqrt{-g}} \frac{\delta S_{\mathrm{mat}}\left[\left\{\phi_{0 i}\right\}, g_{\mu \nu} ;\left\{\lambda_{0 a}\right\}\right]}{\delta g_{\mu \nu}} .
$$

The $d$-dimensional flat-spacetime energy-momentum tensor in the scalar QED is given by

$$
\begin{aligned}
T_{\mu \nu}= & -g^{\lambda \kappa} F_{0 \mu \lambda} F_{0 \nu \kappa}+2 D_{0 \mu} \phi_{0}^{*} D_{0 \nu} \phi_{0} \\
& -2\left(\xi_{c}+\frac{\eta_{0}}{d-1}\right)\left(\partial_{\mu} \partial_{\nu}-g_{\mu \nu} \partial^{2}\right)\left|\phi_{0}\right|^{2} \\
& -g_{\mu \nu}\left(-\frac{1}{4} F_{0 \lambda \kappa}^{2}+\left|D_{0 \mu} \phi_{0}\right|^{2}-m_{0}^{2}\left|\phi_{0}\right|^{2}-\frac{1}{4} \lambda_{0}\left|\phi_{0}\right|^{4}\right) .
\end{aligned}
$$

Taking the trace, one finds

$$
\begin{aligned}
T_{\mu}^{\mu}= & \epsilon\left(-\frac{1}{4} F_{0 \mu \nu}^{2}+\frac{1}{4} \lambda_{0}\left|\phi_{0}\right|^{4}\right)+2 m_{0}^{2}\left|\phi_{0}\right|^{2}+2 \eta_{0} \partial^{2}\left|\phi_{0}\right|^{2} \\
& + \text { (e.o.m.) }
\end{aligned}
$$

where the last term is proportional to the equation of motion as 


$$
\begin{aligned}
(\text { e.o.m. })= & \left(1-\frac{\epsilon}{2}\right) \phi_{0}^{*}\left(D_{0}^{2} \phi_{0}+m_{0}^{2} \phi_{0}+\frac{2}{4} \lambda_{0}\left|\phi_{0}\right|^{2} \phi_{0}\right) \\
& +\left(1-\frac{\epsilon}{2}\right)\left(D_{0}^{2} \phi_{0}^{*}+m_{0}^{2} \phi_{0}^{*}+\frac{2}{4} \lambda_{0}\left|\phi_{0}\right|^{2} \phi_{0}^{*}\right) \phi_{0} .
\end{aligned}
$$

In the flat spacetime, $T^{\mu}{ }_{\mu}$ is almost predetermined by the multiplicative renormalization of the fields and parameters. The single exception is the nonminimal coupling $\eta_{0}$, whose renormalization is determined by the renormalization of $T^{\mu}{ }_{\mu}$ itself. We renormalize $\eta_{0}$ as

$$
Z_{\phi} \eta_{0}=Z_{\eta} \eta
$$

with the wave function renormalization of $\phi$ being $Z_{\phi}^{1 / 2}$. As discussed in Ref. [17], the RGE of $\eta$ takes a form of

$$
\frac{d \eta}{d \ln \mu}=\gamma_{\phi^{2}}^{T} \eta+\tilde{\beta}_{\eta}
$$

where $\eta$ and $\gamma_{\phi^{2}}$ should be understood as a vector and matrix, respectively, for multiple scalar fields. Here

$$
\phi_{0}^{2}=Z_{\phi^{2}}\left[\phi^{2}\right], \quad \frac{d \ln Z_{\phi^{2}}}{d \ln \mu}=\gamma_{\phi^{2}},
$$

and a square bracket denotes the renormalized composite operator. The homogeneous term is proportional to the anomalous dimension of $\phi^{2}$ in the RGE. This is because the renormalization of the scalar field squared is multiplicative, $Z_{\phi^{2}}^{-1} \partial^{2} \phi_{0}^{2}=\partial^{2}\left[\phi^{2}\right]$. It means that all the counterterms to renormalize $\phi^{2}$ is included in $Z_{\phi^{2}}^{-1} Z_{\phi}$. $\tilde{\beta}_{\eta}$ denotes the inhomogeneous term of the RGE and induces $\Delta \eta$ through the running irrespective of our initial choice of $\eta$. This $\Delta \eta$ is nothing but a quantum-induced value of $\eta$.

In the following, we evaluate the renormalization of $\eta$, i.e., the finite correction and RGE, in the scalar QED. We take the Feynman-'t Hooft gauge, but note that $\eta$ is a gauge-invariant quantity and thus its RGE does not depend on the gauge choice. For diagrammatic convenience, we introduce the "scalaron" $\sigma$ that couples to $T^{\mu}{ }_{\mu}$ as

$$
\mathcal{L}_{\sigma \text {-mat }}=\sigma T^{\mu}{ }_{\mu}
$$

We calculate the amputated amplitude of scalaron decay into light scalars: $\sigma(p) \rightarrow \phi^{*}(q) \phi(k)$, where $p, q$, and $k$ are external momenta. Although we consider scalaron decay, our analysis is applicable even to studying the properties of nonminimal couplings in the models without the scalaron.

The leading contributions to the scalaron decay originate from

$$
\begin{aligned}
T_{\mu}^{\mu} \supset & -\frac{1}{4} \epsilon F_{\mu \nu}^{2}+2 m^{2}|\phi|^{2}+2 \eta \partial^{2}|\phi|^{2}-\frac{1}{4} \epsilon\left(Z_{A}-1\right) F_{\mu \nu}^{2} \\
& +2\left(Z_{m^{2}}-1\right) m^{2}|\phi|^{2}+2\left(Z_{\eta}-1\right) \eta \partial^{2}|\phi|^{2}
\end{aligned}
$$

where we use the renormalized fields and couplings. Hereafter we assume that the quartic coupling $\lambda$ is negligible. As stressed in Ref. [53], a key point is that $T^{\mu}{ }_{\mu}$ contains terms proportional to $\epsilon$ : $T^{\mu}{ }_{\mu} \supset-(1 / 4) \epsilon F_{0 \mu \nu}^{2}$. These terms vanish in the limit of $\epsilon \rightarrow 0$ at the classical level, but not at the quantum level due to the divergent corrections to composite operators: $F_{0 \mu \nu}^{2}$. This is the origin of the trace anomaly.

\section{ONE-LOOP ORDER}

Figure 1 shows one-loop diagrams contributing to $\sigma(p) \rightarrow \phi^{*}(q) \phi(k)$ in the scalar QED. We summarize one-loop calculations in Appendix B. Figure 1(a) originates from the insertion of $T^{\mu}{ }_{\mu} \supset 2 m^{2}|\phi|^{2}+2 \eta \partial^{2}|\phi|^{2}$. The divergent part [see Eq. (B12) for the full expression] is

$$
\left(i \mathcal{M}_{(a)}^{\text {loop }}\right)_{\text {of } \epsilon}^{\text {pole }}=4 i\left(m^{2}-\eta p^{2}\right) \frac{Q^{2} e^{2}}{16 \pi^{2}} \frac{1}{\epsilon} .
$$

Figures 1(b) and 1(c) originate from the insertion of $T^{\mu}{ }_{\mu} \supset$ $-(1 / 4) \epsilon F_{\mu \nu}^{2}$ and provide only finite contributions:

$i \mathcal{M}_{(b)}^{\text {loop }}=3 i \frac{Q^{2} e^{2}}{16 \pi^{2}} p^{2}, \quad i \mathcal{M}_{(c)}^{\text {loop }}=-6 i \frac{Q^{2} e^{2}}{16 \pi^{2}}(k \cdot q)$.

In other words, the inhomogeneous term of the RGE $\tilde{\beta}_{\eta}$ does not arise from the trace anomaly at the one-loop order. These finite contributions are proportional to the scalaron momentum squared $p^{2}$. They would be the finite correction to $\eta$, since the diagrams from the insertion of $T^{\mu}{ }_{\mu} \supset 2 \eta \partial^{2}|\phi|^{2}$ are also proportional to $p^{2}$. However, when the contributions are summed up, the term proportional to $p^{2}$ vanishes,

$$
i \mathcal{M}_{(b)+(c)}^{\mathrm{loop}}=6 i \frac{Q^{2} e^{2}}{16 \pi^{2}} m^{2} .
$$

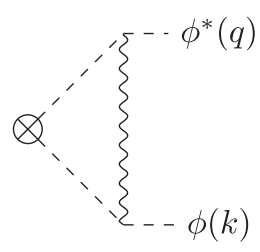

(a)

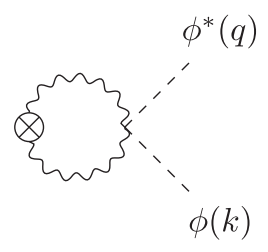

(b)

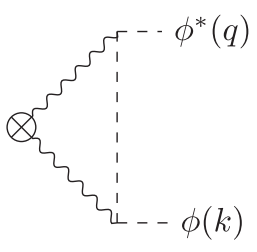

(c)
FIG. 1. One-loop diagrams for scalaron decay $\sigma(p) \rightarrow$ $\phi^{*}(q) \phi(k)$. Crossed dots denote insertion of the energymomentum tensor, (a) $2 m^{2}|\phi|^{2}+2 \eta \partial^{2}|\phi|^{2}$, and (b), (c) $-(1 / 4) \epsilon F_{\mu \nu}^{2}$. 
This contribution is reproduced by the insertion of $-\beta_{m^{2}}|\phi|^{2}$, where $\beta_{m^{2}}$ is the $\beta$ function of $m^{2}$ in Eq. (B10). Meanwhile, there is no contribution proportional to $p^{2}$, which can be regarded as a finite value of $\eta$.

The divergence of $i \mathcal{M}_{(a)}^{\text {loop }}$ is canceled by the counterterm contributions of $T_{\mu}^{\mu} \supset 2\left(Z_{m^{2}}-1\right) m^{2}|\phi|^{2}+2\left(Z_{\eta}-1\right) \eta \partial^{2}|\phi|^{2}$. We again note that $Z_{m^{2}}-1$ is predetermined by the self-energy of $\phi$ [see Eq. (B8)]. The counterterm of the nonminimal coupling is determined to absorb this divergence as

$$
Z_{\eta}-1=-2 \frac{Q^{2} e^{2}}{16 \pi^{2}} \frac{1}{\epsilon} .
$$

From $Z_{\phi}$ in Eq. (B8), one obtains

$$
\frac{d \eta}{d \ln \mu}=-6 \frac{Q^{2} e^{2}}{16 \pi^{2}} \eta
$$

at the one-loop level. At this order, we only find a homogeneous term of the RGE and we also confirm the expression of Eq. (8) from Eq. (B22). The solution is

$$
\eta=\eta_{i}\left(\frac{e^{2}}{e_{i}^{2}}\right)^{-9} .
$$

Here, the subscript $i$ denotes the boundary condition for the RGE: $\eta=\eta_{i}$ at $e=e_{i} . \eta$ can deviate from its initial value $\eta_{i}$ through the homogeneous term of the RGE. Since the scalar QED becomes more weakly coupled toward a lower energy, the low-energy $\eta$ grows from $\eta_{i}$. However, to be consistent with the perturbative analysis, the low-energy $\eta$ does not change significantly even though the exponent of the solution is large. For example, the homogeneous solution Eq. (17) gets larger by an order of magnitude as the QED coupling $e^{2}$ gets smaller by about $30 \%$ than $e_{i}^{2}$. If $e^{2}$ changes by an order of magnitude, we may need to incorporate next-leading or higher order corrections in $e^{2}$.

Note that an important quantity for the inflation is $\xi$ not $\eta$. This solution of the RGE provides the nonminimal coupling $\xi$ at the low energy in the scalar QED,

$\xi=\xi_{i}\left(\frac{e^{2}}{e_{i}^{2}}\right)^{-9}+\frac{1}{6}\left[1-\left(\frac{e^{2}}{e_{i}^{2}}\right)^{-9}\right]+\frac{1}{3}\left[\tilde{\eta}-\tilde{\eta}_{i}\left(\frac{e^{2}}{e_{i}^{2}}\right)^{-9}\right]$.

The first and second terms originate from the homogeneous solution of $\eta$, while the third term would originate from the inhomogeneous solution of $\eta(\Delta \eta) . \tilde{\eta}$ is the inhomogeneous solution of Eq. (8), whose initial value is denoted by $\tilde{\eta}_{i}$. Since we find $\gamma_{\phi^{2}}$ at the one-loop order, if we found $n$-loop order $\tilde{\beta}_{\eta}, \tilde{\eta}$ would be at the $(n-1)$-loop level. Note that the deviation of $\tilde{\eta}$ from $\tilde{\eta}_{i}\left(e^{2} / e_{i}^{2}\right)^{-9}$ is at the next loop level to $\tilde{\eta}$ itself; i.e., an $(n-1)$-loop order $\tilde{\eta}$ gives an $n$-loop order contribution to the inhomogeneous term of $\xi(\Delta \eta / 3)$. Since we find no one-loop order $\tilde{\beta}_{\eta}$, and there is neither $\tilde{\eta}$ at the tree level nor the one-loop order contribution to the inhomogeneous term of $\xi$.

We note that the above discussion does not change for the scalar QCD. In the scalar QCD, $Q^{2}$ is replaced by $T(S)$ denoting the one-half of the Dynkin index of the representation for scalar fields. The analytic solution Eq. (17) depends on the $\beta$ function of $e$ at the one-loop level, and thus the power of the coupling $e$ in Eq. (17) will change in the scalar QCD.

\section{TWO-LOOP ORDER}

We focus only on the RGE of $\eta$, which is determined by the divergent part of the diagrams. Contributions to the nonminimal coupling are proportional to an incoming momentum squared $p^{2}$. Thus, we take the massless limit of $\phi: m \rightarrow 0$. We also focus on the inhomogeneous term of the RGE $\tilde{\beta}_{\eta}$, which originates from the insertion of $T^{\mu}{ }_{\mu} \supset-(1 / 4) \epsilon F_{0 \mu \nu}^{2}$.

There are two types of two-loop contributions from the insertion of $T^{\mu}{ }_{\mu} \supset-(1 / 4) \epsilon F_{0 \mu \nu}^{2}$ : (1) one-loop diagrams with the insertion of $T^{\mu}{ }_{\mu} \supset\left(\beta_{e} / 2 e\right) F_{\mu \nu}^{2}$; and (2) two-loop order diagrams with the insertion of $T^{\mu}{ }_{\mu} \supset-(1 / 4) \epsilon F_{\mu \nu}^{2}$. Here $\beta_{e}$ is the one-loop $\beta$ function of $e$ [see Eq. (B4)], which is obtained from $\epsilon\left(Z_{A}-1\right)=-2 \beta_{e} / e$ at the oneloop level. We remark that this simple relation between $\beta_{e}$ and $Z_{A}$ holds only in the QED because of the WardTakahashi identity. In the QCD, to obtain the $\beta$ function from $T^{\mu}{ }_{\mu} \supset-(1 / 4) \epsilon F_{0 \mu \nu}^{2}$, one has to take into account selfcouplings of the gauge boson. We demonstrate it in Appendix $\mathrm{D}$, since intriguingly it gives another derivation of the one-loop $\beta$ function in the QCD.

First, we consider the type (1) contributions. They are given by Figs. 1(b) and 1(c) with the insertion of $T^{\mu}{ }_{\mu} \supset$ $\left(\beta_{e} / 2 e\right) F_{\mu \nu}^{2}$ instead of $T^{\mu}{ }_{\mu} \supset-(1 / 4) \epsilon F_{\mu \nu}^{2}$. The divergent parts are

$$
\begin{aligned}
& \left(i \mathcal{M}_{(b)}^{1 \text {-loop }}\right)_{\text {of } \epsilon}^{\text {pole }}=-\frac{2 \beta_{e}}{e} \frac{1}{\epsilon} i \mathcal{M}_{(b)}^{\text {loop }}, \\
& \left(i \mathcal{M}_{(c)}^{1 \text { loop }}\right)_{\text {of } \epsilon}^{\text {pole }}=-\frac{2 \beta_{e}}{e} \frac{1}{\epsilon} i \mathcal{M}_{(c)}^{\text {loop }},
\end{aligned}
$$

where $i \mathcal{M}_{(b)}^{\text {loop }}$ and $i \mathcal{M}_{(c)}^{\text {loop }}$ are shown in Fig. 1 and given by Eq. (13). As before [Eq. (14)], the terms proportional to $p^{2}$ cancel with each other. Thus, there is no contribution to $\tilde{\beta}_{\eta}$.

Next, we consider the type (2) contributions. They are given by Fig. 2 with the insertion of $T^{\mu}{ }_{\mu} \supset-(1 / 4) \epsilon F_{\mu \nu}^{2}$. For 2(a1)-2(a4) and 2(c1)-2(c4), there are contributions also from diagrams with internal photons being exchanged. For 2(a1) and 2(a3), there are also contributions from the $A_{\mu}^{2}|\phi|^{2}$ vertex, but they vanish in the massless limit of $m \rightarrow 0$ in the dimensional regularization. There are two types of diagrams among the type (2) contributions: twoloop diagrams without the insertion of counterterms, and 

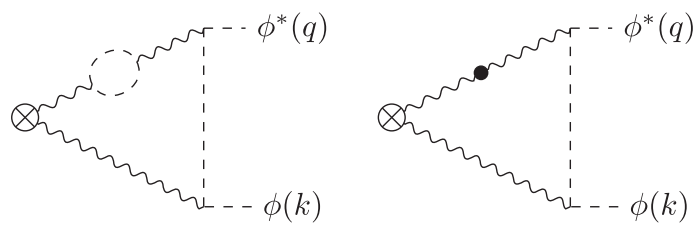

(a1)

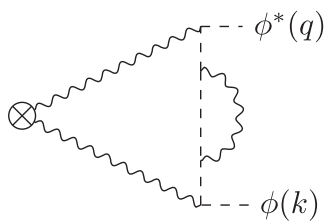

(b1)

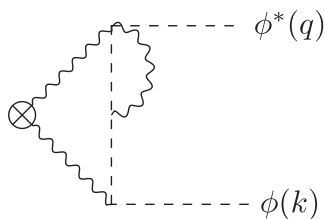

(c3)

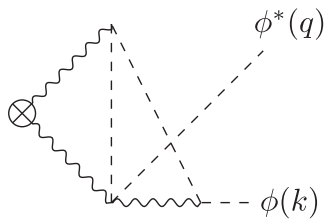

(d2')

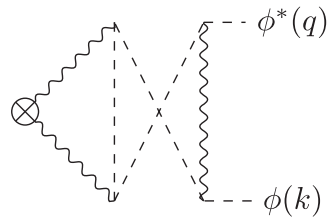

(d4')

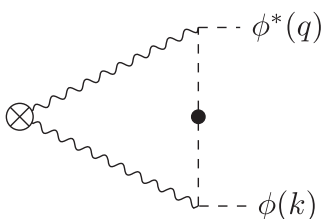

(b2)

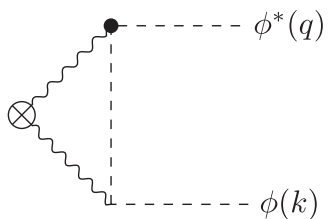

(c4)

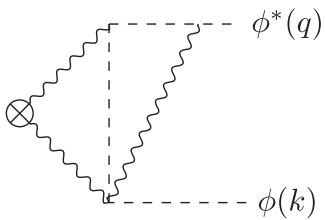

(d3)

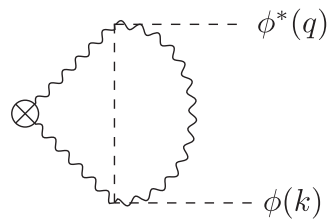

(d5)

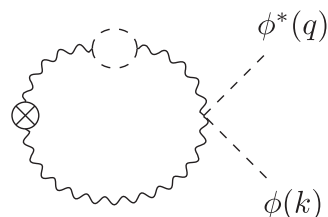

(a3)

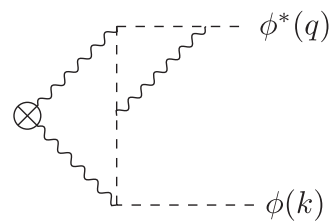

(c1)

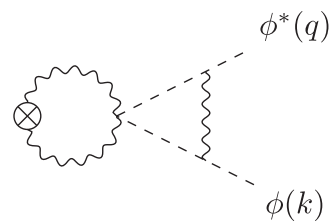

(d1)

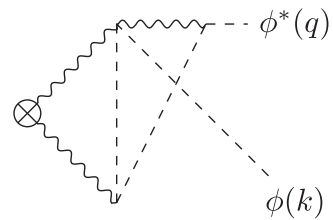

(d3')

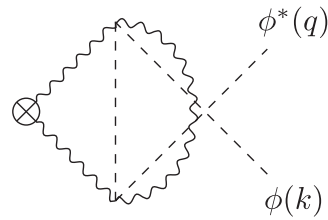

(d5')

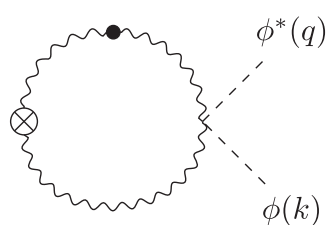

(a4)

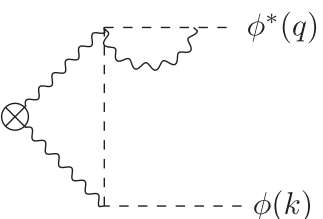

(c2)

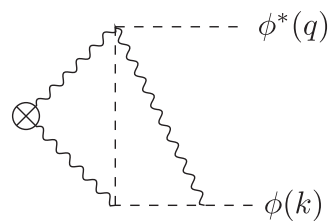

(d2)

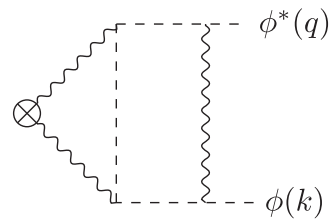

(d4)

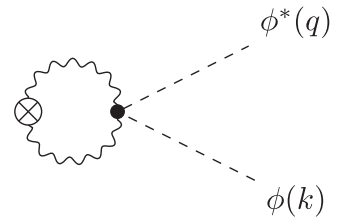

(d6)

FIG. 2. Two-loop order diagrams for scalaron decay $\sigma(p) \rightarrow \phi^{*}(q) \phi(k)$. For (a1)-(a4) and (c1)-(c4), there are contributions also from diagrams with internal photons being exchanged. For (a1) and (a3), there are also contributions from the $A_{\mu}^{2}|\phi|^{2}$ vertex, but they vanish in the massless limit of $m \rightarrow 0$ in the dimensional regularization. Crossed dots denote the insertion of the energy-momentum tensor, $-(1 / 4) \epsilon F_{\mu \nu}^{2}$. Filled dots denote the insertion of the counterterms: (a2), (a4) $-(1 / 4)\left(Z_{A}-1\right) F_{\mu \nu}^{2},(\mathrm{~b} 2)\left(Z_{\phi}-1\right)\left|\partial_{\mu} \phi\right|^{2},(\mathrm{c} 4)$ $i Q\left(Z_{e}-1\right) \tilde{\mu}^{\epsilon / 2} e A^{\mu}\left(\phi^{*} \partial_{\mu} \phi-\partial_{\mu} \phi^{*} \phi\right)$, and (d6) $Q^{2}\left(Z_{e^{2}}-1\right) \tilde{\mu}^{\epsilon} e^{2} A_{\mu}^{2}|\phi|^{2}$.

one-loop diagrams with the insertion of one-loop counterterms (black dots in Fig. 2). Though direct evaluation of two-loop diagrams is beyond the scope of this paper, we evaluate the $1 / \epsilon$ pole [i.e., $(1 / \epsilon)^{2}$ pole except for $\epsilon$ from $\left.-(1 / 4) \epsilon F_{\mu \nu}^{2}\right]$ based on the finiteness of renormalizable theory such as the scalar QED (concretely, renormalizability of the composite operator, $T_{\mu}^{\mu} \supset F_{\mu \nu}^{2}$ ) in light of the Bogoliubov-Parasiuk-Hepp-Zimmermann (BPHZ) theorem $[6,54,55]$. In the dimensional regularization, two-loop diagrams give the leading divergence of $\left.i \mathcal{M}^{2 \text {-loop }}\right|_{\text {div }} ^{\text {leading }}=i \epsilon p^{2}\left(\frac{Q^{2} e^{2}}{16 \pi^{2}} \frac{1}{\epsilon}\right)^{2} \mu^{2 \epsilon} F^{2-\operatorname{loop}}(p, q, k)$

where $F^{2 \text {-loop }}(p, q, k)$ is finite. Meanwhile, one-loop diagrams with the insertion of counterterms give

$\left.i \mathcal{M}^{1 \text {-loop }}\right|_{\mathrm{div}}=i \epsilon p^{2}\left(\frac{Q^{2} e^{2}}{16 \pi^{2}} \frac{1}{\epsilon}\right)\left(\frac{Q^{2} e^{2}}{16 \pi^{2}} \frac{1}{\epsilon}\right) \mu^{\epsilon} F^{1-\mathrm{loop}}(p, q, k)$, 
where $F^{1-\text { loop }}(p, q, k)$ is finite. The former $\left(Q^{2} e^{2} / 16 \pi^{2}\right) \times$ $(1 / \epsilon)$ comes from the one-loop counterterm, and the latter $\left(Q^{2} e^{2} / 16 \pi^{2}\right)(1 / \epsilon) \mu^{\epsilon}$ comes from the one-loop diagram. The finiteness of the theory means that there are no nonlocal divergences and thus no $\epsilon(1 / \epsilon) \ln \mu$ relating numerical coefficients of the above two contributions:

$$
2 F^{2 \text {-loop }}+F^{1 \text {-loop }}=0 .
$$

Thus, we only need to evaluate one-loop diagrams to

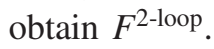

In Appendix C, we take a closer look at two-loop order diagrams and their subdivergences. The one-loop diagrams with the insertion of counterterms give

$$
\begin{aligned}
& i \mathcal{M}_{(a 2)}^{1 \text {-loop }}=-2\left(Z_{A}-1\right) i \mathcal{M}_{(c)}^{\text {loop }}, \\
& i \mathcal{M}_{(a 4)}^{1-\text { loop }}=-2\left(Z_{A}-1\right) i \mathcal{M}_{(b)}^{\text {loop }}, \\
& i \mathcal{M}_{(b 2)}^{1 \text { loop }}=-\left(Z_{\phi}-1\right) i \mathcal{M}_{(c)}^{\text {loop }}, \\
& i \mathcal{M}_{(c 4)}^{1 \text { loop }}=2\left(Z_{e}-1\right) i \mathcal{M}_{(c)}^{\text {loop }}, \\
& i \mathcal{M}_{(d 6)}^{1 \text { loop }}=\left(Z_{e^{2}}-1\right) i \mathcal{M}_{(b)}^{\text {loop }},
\end{aligned}
$$

where $i \mathcal{M}_{(b)}^{\text {loop }}$ and $i \mathcal{M}_{(c)}^{\text {loop }}$ are shown in Fig. 1 and given by Eq. (13). Noting the Ward-Takahashi identity, $Z_{\phi}=Z_{e}=Z_{e^{2}}$, and also that $i \mathcal{M}_{(b)+(c)}^{\text {loop }}$ does not give any term proportional to $p^{2}$, we find that $F^{1 \text {-loop }}=0$ and thus $F^{2 \text {-loop }}=0$. In summary, there is no contribution to $\tilde{\beta}_{\eta}$.

The above discussion is not directly applicable to the massless scalar QCD. There are more diagrams appearing from the self-couplings of the gauge boson. On the other hand, we would expect that there is no contribution to $\tilde{\beta}_{\eta}$ at the two-loop level even in the massless scalar QCD. We discuss the physical reason in the next section. Though it is beyond the scope of this paper, it will be important to note the following to see it in a similar calculation to this section: the Slavnov-Taylor identity [56,57] gives $\left(Z_{e^{2}}-1\right)=2\left(Z_{e}^{2}-1\right)-\left(Z_{\phi}-1\right)$ at the one-loop level; and $i \mathcal{M}_{(b)+(c)}^{\text {loop }}$ does not provide the $p^{2}$ term at the one-loop level as seen in the previous section.

\section{DISCUSSION}

The energy-momentum tensor is the single (and thus valuable) place where we can study the properties of a nonminimal coupling in the flat spacetime. We have studied the RGE of $\eta$ in the scalar QED at the two-loop level, through the renormalization of $T^{\mu}{ }_{\mu}$.

We have found a homogeneous term of the RGE at the one-loop order. In the scalar QED, the homogeneous solution of the RGE blows up toward a low energy as the gauge theory becomes more weakly coupled. This is because of $\gamma_{\phi^{2}}<0$, in contrast to the $\lambda \phi^{4}$ theory [3-5] and Yukawa theory [17]. Since the scalar mass squared follows $\beta_{m^{2}}=\gamma_{\phi^{2}} m^{2}$, the scalar mass squared also blows up toward a low energy. It means that the scalar QED does not approach a massless (conformal) free scalar theory at a low energy, without fine-tuning.

On the other hand, we have found neither the finite correction to $\eta$ nor the inhomogeneous term of the RGE of $\eta$ at the one-loop order as in the $\lambda \phi^{4}$ theory [3-5]. Since we have found no finite correction to $\eta$ at the one-loop order, it is expected that there is also no inhomogeneous term of the RGE of $\eta$ at the two-loop order. We have explicitly confirmed it. We note that there could be the two-loop order finite correction to $\eta$, which we leave for future work.

We may expect this result in a generic class of theories for the following reasoning. For the usual quantities without any reason, we expect the inhomogeneous solution of the RGE at the one-loop order. For example, in the scalar $\mathrm{QCD}$, the quartic coupling (which we assume is zero) is induced by the gauge coupling at the one-loop order (though it does not change the above discussion). In contrast, we may expect the inhomogeneous solution of the nonminimal coupling $\eta$ at the two-loop order or higher. The inhomogeneous solution of $\eta$ is expected to originate from the quantum breaking of scale invariance, i.e., the trace anomaly that appears only at the loop order. It means that an extra loop order or higher is required to induce $\eta$ only from the usual couplings (such as gauge, Yukawa, and quartic couplings). We will check this expectation, i.e., no inhomogeneous term of the RGE at the two-loop order, in the two-scalar theory, Yukawa theory, and scalar QCD, somewhere else.

Now, we discuss the implication of the nonminimal coupling for inflationary models. The predictions of the inflationary models can change in the presence of the nonminimal coupling even of the order of $10^{-3}$. For instance, the chaotic inflation models with a power-law potential are still viable when an inflaton field has the nonminimal coupling of $10^{-3} \cdot \xi \sim-10^{-3}$ is naturally expected to arise from quantum corrections, in particular, at the one-loop order. We have studied the RGE of $\eta$ in the scalar QED, and have found no inhomogeneous term of the RGE even at the two-loop order. As discussed above, we expect that there is no inhomogeneous term of the RGE of $\eta$ at the two-loop order in various theories. Thus, it is enough to consider the homogeneous term of the RGE of $\eta$, which accounts for a part of the inhomogeneous term of the RGE of $\xi$, to obtain $\xi$ of $10^{-3}$. One can also consider the decoupling effect of the heavy field besides the RGE to get $\xi$ of the one-loop order as a quantum-induced value [17]. The decoupling of the heavy fields gives a threshold correction to $\xi$ at the one-loop level, irrespective of our initial choice of $\xi$. 
Finally we remark that there is one important subtlety when one identifies $\phi$ as the inflaton. During inflation, $\phi$ may take a field value, which breaks the gauge invariance and gives mass to gauge bosons. In this case, an additional contribution to the threshold correction may appear from diagrams with the gauge boson mass term being inserted. We will also study it somewhere else.

\section{ACKNOWLEDGMENTS}

The work of A. K. and T. K. is supported by IBS under Project No. IBS-R018-D1. A. K. thanks Kazuya Yonekura for valuable advice.

\section{APPENDIX A: SCALAR QED}

We use the $\overline{\mathrm{MS}}$ scheme with the spacetime dimensions of $d=4-\epsilon$ and the renormalization scale $\mu$. We compensate a mass dimension by the modified renormalization scale $\tilde{\mu}$, which is defined as

$$
\tilde{\mu}^{2}=\mu^{2} \frac{e^{\gamma_{E}}}{4 \pi}
$$

with $\gamma_{E} \simeq 0.577$ being Euler's constant. We use Feynman't Hooft gauge $\left(\xi^{g f}=1\right)$ in the loop calculations.
The Lagrangian density is

$$
\begin{aligned}
\mathcal{L}= & -\frac{1}{4} F_{0 \mu \nu}^{2}-\frac{1}{2 \xi_{0}^{\mathrm{gf}}}\left(\partial_{\mu} A_{0}^{\mu}\right)^{2}+\left|D_{0 \mu} \phi_{0}\right|^{2}-m_{0}^{2}\left|\phi_{0}\right|^{2} \\
& -\frac{1}{4} \lambda_{0}\left|\phi_{0}\right|^{4},
\end{aligned}
$$

with $D_{0 \mu}=\partial_{\mu}-i Q e_{0} A_{0 \mu}$ being the gauge covariant derivative for a charge $Q$. We have integrated out the Nakanishi-Lautrup field and (anti)ghost fields.

The multiplicative renormalization is set for fields as

$$
\phi_{0}=Z_{\phi}^{1 / 2} \phi, \quad A_{0 \mu}=Z_{A}^{1 / 2} A_{\mu}
$$

and for parameters as

$$
\begin{aligned}
& Z_{\phi} Z_{A}^{1 / 2} e_{0}=Z_{e} \tilde{\mu}^{\epsilon / 2} e, Z_{\phi} Z_{A} e_{0}^{2}=Z_{e^{2}} \tilde{\mu}^{\epsilon} e^{2} \text { (i.e., } Z_{\phi} Z_{e^{2}}=Z_{e}^{2} \text { ), } \\
& Z_{\phi} m_{0}^{2}=Z_{m^{2}} m^{2}, \quad Z_{\phi}^{2} \lambda_{0}=Z_{\lambda} \tilde{\mu}^{\epsilon} \lambda, \quad Z_{A} / \xi_{0}^{\mathrm{gf}}=Z_{\xi^{\mathrm{gf}}} / \xi^{\xi^{\mathrm{gf}}},
\end{aligned}
$$

and

$$
Z_{\phi} \eta_{0}=Z_{\eta} \eta
$$

The Lagrangian density in terms of the renormalized quantities is

$$
\begin{aligned}
\mathcal{L}= & -\frac{1}{4} F_{\mu \nu}^{2}-\frac{1}{2 \xi^{\mathrm{gf}}}\left(\partial_{\mu} A^{\mu}\right)^{2}+\left|\partial_{\mu} \phi\right|^{2}-m^{2}|\phi|^{2}+i Q \tilde{\mu}^{\epsilon / 2} e A^{\mu}\left(\phi^{*} \partial_{\mu} \phi-\partial_{\mu} \phi^{*} \phi\right)+Q^{2} \tilde{\mu}^{\epsilon} e^{2} A_{\mu}^{2}|\phi|^{2}-\frac{1}{4} \tilde{\mu}^{\epsilon} \lambda|\phi|^{4} \\
& -\frac{1}{4}\left(Z_{A}-1\right) F_{\mu \nu}^{2}-\frac{1}{2 \xi^{\mathrm{gf}}}\left(Z_{\xi^{\mathrm{gf}}}-1\right)\left(\partial_{\mu} A^{\mu}\right)^{2}+\left(Z_{\phi}-1\right)\left|\partial_{\mu} \phi\right|^{2}-\left(Z_{m^{2}}-1\right) m^{2}|\phi|^{2} \\
& +i Q\left(Z_{e}-1\right) \tilde{\mu}^{\epsilon / 2} e A^{\mu}\left(\phi^{*} \partial_{\mu} \phi-\partial_{\mu} \phi^{*} \phi\right)+Q^{2}\left(Z_{e^{2}}-1\right) \tilde{\mu}^{\epsilon} e^{2} A_{\mu}^{2}|\phi|^{2}-\frac{1}{4}\left(Z_{\lambda}-1\right) \tilde{\mu}^{\epsilon} \lambda|\phi|^{4} .
\end{aligned}
$$

The Ward-Takahashi identity warrants that $Z_{e}=Z_{\phi}=Z_{e^{2}}, Z_{A}$ is independent of $\xi^{\mathrm{gf}}, Z_{\xi^{\mathrm{gf}}}=1$. It follows that

$$
\begin{aligned}
\beta_{e}^{\epsilon} & =e\left(-\frac{1}{2} \epsilon+\frac{1}{2} \frac{d \ln Z_{A}}{d \ln \mu}\right), \\
\beta_{\lambda}^{\epsilon} & =\lambda\left(-\epsilon+2 \frac{d \ln Z_{\phi}}{d \ln \mu}-\frac{d \ln Z_{\lambda}}{d \ln \mu}\right), \\
\beta_{m^{2}} & =m^{2}\left(\frac{d \ln Z_{\phi}}{d \ln \mu}-\frac{d \ln Z_{m^{2}}}{d \ln \mu}\right), \\
\beta_{\xi^{g f}} & =-\xi^{\mathrm{gf}} \frac{d \ln Z_{A}}{d \ln \mu}, \\
\beta_{\eta} & =\eta\left(\frac{d \ln Z_{\phi}}{d \ln \mu}-\frac{d \ln Z_{\eta}}{d \ln \mu}\right) .
\end{aligned}
$$




\section{APPENDIX B: ONE-LOOP ORDER IN THE SCALAR QED}

One-loop functions are summarized in Appendix B. 1. The arguments of the one-loop functions are omitted when they are obvious. The results here are applicable to the scalar QCD by replacing $Q^{2}$ by $T(S)$ denoting the one-half of the Dynkin index of the representation for scalar fields.

The one-loop self-energy of the gauge boson $A_{\mu}$ is given by

$$
\begin{aligned}
i \Pi^{\mu \nu}(p) & =\left(i Q e \tilde{\mu}^{\epsilon / 2}\right)^{2} \int \frac{d^{d} \ell}{(2 \pi)^{d}} \frac{i(2 \ell+p)^{\mu} i(2 \ell+p)^{\nu}}{\left[\ell^{2}-m^{2}\right]\left[(\ell+p)^{2}-m^{2}\right]}+\left(2 i Q^{2} e^{2} g^{\mu \nu} \tilde{\mu}^{\epsilon}\right) \int \frac{d^{d} \ell}{(2 \pi)^{d}} \frac{i}{\left[\ell^{2}-m^{2}\right]} \\
& =i \frac{Q^{2} e^{2}}{16 \pi^{2}}\left[\left(4 B_{22}-2 A\right) g^{\mu \nu}+\left(4 B_{21}+4 B_{1}+B_{0}\right) p^{\mu} p^{\nu}\right] \\
& =i\left(p^{2} g^{\mu \nu}-p^{\mu} p^{\nu}\right) \frac{Q^{2} e^{2}}{16 \pi^{2}} \frac{4}{3 p^{2}}\left[-A+\left(m^{2}-\frac{p^{2}}{4}\right) B_{0}+m^{2}-\frac{p^{2}}{6}\right] .
\end{aligned}
$$

The counterterm is determined to make the vacuum polarization finite,

$$
\left(i \Pi^{\mu \nu}\right)^{\text {c.t. }}(p)=-i\left(Z_{A}-1\right)\left(p^{2} g^{\mu \nu}-p^{\mu} p^{\nu}\right)
$$

and we obtain

$$
Z_{A}-1=-\frac{2}{3} \frac{Q^{2} e^{2}}{16 \pi^{2}} \frac{1}{\epsilon}
$$

The $\beta$ function $\beta_{e}$ at the one-loop level is

$$
\beta_{e}=\frac{1}{3} \frac{Q^{2} e^{3}}{16 \pi^{2}}
$$

The resultant self-energy is

$$
\begin{aligned}
\Gamma_{2}^{\mu \nu}(p)= & -\left(p^{2} g^{\mu \nu}-p^{\mu} p^{\nu}\right)\left[1+\frac{Q^{2} e^{2}}{16 \pi^{2}} \frac{4}{3 p^{2}}\left(m^{2} \int_{0}^{1} d x \ln \left(\frac{m^{2}-x(1-x) p^{2}}{m^{2}}\right)\right.\right. \\
& \left.\left.-\frac{p^{2}}{4} \int_{0}^{1} d x \ln \left(\frac{m^{2}-x(1-x) p^{2}}{\mu^{2}}\right)+\frac{p^{2}}{6}\right)\right] .
\end{aligned}
$$

The one-loop self-energy of the scalar field $\phi$ is given by

$$
\begin{aligned}
i \Pi\left(p^{2}\right) & =\left(i Q e \tilde{\mu}^{\epsilon / 2}\right)^{2} \int \frac{d^{d} \ell}{(2 \pi)^{d}} \frac{-i g_{\mu \nu}}{\ell^{2}} \frac{(\ell+2 p)^{\mu} i(\ell+2 p)^{\nu}}{(\ell+p)^{2}-m^{2}}+\left(2 i Q^{2} e^{2} g^{\mu \nu} \tilde{\mu}^{\epsilon}\right) \frac{1}{2} \int \frac{d^{d} \ell}{(2 \pi)^{d}} \frac{-i g_{\mu \nu}}{\ell^{2}} \\
& =i \frac{Q^{2} e^{2}}{16 \pi^{2}}\left[(d-1) A\left(0^{2}\right)-2 p^{2} B_{1}\left(p^{2} ; 0^{2}, m^{2}\right)-\left(3 p^{2}+m^{2}\right) B_{0}\right] \\
& =i \frac{Q^{2} e^{2}}{16 \pi^{2}}\left[A\left(m^{2}\right)-2\left(p^{2}+m^{2}\right) B_{0}\right] .
\end{aligned}
$$

In the first equality, we take into account the symmetric factor of $1 / 2$. In the second equality, we use $g_{\mu \nu}(\ell+2 p)^{\mu}(\ell+2 p)^{\nu}=\left[(\ell+p)^{2}-m^{2}\right]+2 \ell \cdot p+3 p^{2}+m^{2}$. The counterterm is determined to make the vacuum polarization finite,

$$
i \Pi^{\text {c.t. }}\left(p^{2}\right)=-i\left(Z_{m^{2}}-1\right) m^{2}+i\left(Z_{\phi}-1\right) p^{2},
$$

and we obtain 


$$
Z_{m^{2}}-1=-2 \frac{Q^{2} e^{2}}{16 \pi^{2}} \frac{1}{\epsilon}, \quad Z_{\phi}-1=4 \frac{Q^{2} e^{2}}{16 \pi^{2}} \frac{1}{\epsilon} .
$$

The anomalous dimension of $\phi$ is

$$
\gamma_{\phi} \equiv Z_{\phi}^{-1} \frac{d Z_{\phi}}{d \ln \mu}=-4 \frac{Q^{2} e^{2}}{16 \pi^{2}}
$$

One obtains

$$
\beta_{m^{2}}=-6 m^{2} \frac{Q^{2} e^{2}}{16 \pi^{2}}
$$

The resultant self-energy is

$$
\Gamma_{2}\left(p^{2}\right)=p^{2}-m^{2}+\frac{Q^{2} e^{2}}{16 \pi^{2}}\left[m^{2}\left(-\int_{0}^{1} d x \ln \left(\frac{m^{2}}{\mu^{2}}\right)+1\right)+2\left(p^{2}+m^{2}\right) \int_{0}^{1} d x \ln \left(\frac{x m^{2}-x(1-x) p^{2}}{\mu^{2}}\right)\right] .
$$

Figure 1 shows one-loop diagrams contributing to $\sigma(p) \rightarrow \phi^{*}(q) \phi(k)$ in the scalar QED. Figure 1(a) gives

$$
\begin{aligned}
i \mathcal{M}_{(a)}^{\text {loop }} & =2 i\left(m^{2}-\eta p^{2}\right)\left(i Q e \tilde{\mu}^{\epsilon / 2}\right)^{2} \int \frac{d^{d} \ell}{(2 \pi)^{d}} \frac{i(\ell-k)^{\nu}}{\ell^{2}-m^{2}} \frac{-i g_{\mu \nu}}{(\ell+k)^{2}} \frac{i(\ell+q+p)^{\mu}}{(\ell+p)^{2}-m^{2}} \\
& =2 i\left(m^{2}-\eta p^{2}\right) \frac{Q^{2} e^{2}}{16 \pi^{2}}\left[B_{0}\left(p^{2} ; m^{2}, m^{2}\right)+2(q-k)^{\mu} C_{\mu}\left(k^{2}, q^{2}, p^{2} ; m^{2}, 0, m^{2}\right)-2 k \cdot(q+k) C_{0}\right] \\
& =2 i\left(m^{2}-\eta p^{2}\right) \frac{Q^{2} e^{2}}{16 \pi^{2}}\left[B_{0}\left(k^{2} ; m^{2}, 0^{2}\right)+B_{0}\left(q^{2} ; 0^{2}, m^{2}\right)-B_{0}\left(p^{2} ; m^{2}, m^{2}\right)-\left(2 p^{2}-k^{2}-q^{2}-2 m^{2}\right) C_{0}\right] .
\end{aligned}
$$

In the second equality, we use $(\ell-k) \cdot(\ell+p+q)=(\ell+k)^{2}+2 \ell \cdot(q-k)-2 k \cdot(q+k)$.

Figure 1(b) gives

$$
\begin{aligned}
i \mathcal{M}_{(b)}^{\text {loop }} & =i \epsilon\left(2 i Q^{2} e^{2} \tilde{\mu}^{e}\right) \frac{1}{2} \int \frac{d^{d} \ell}{(2 \pi)^{d}}\left[-\ell \cdot(\ell+p) g^{\mu \nu}+\ell^{\mu}(\ell+p)^{\nu}\right] \frac{-i g_{\mu \rho}}{\ell^{2}} \frac{-i g_{\nu \sigma}}{(\ell+p)^{2}} g^{\rho \sigma} \\
& =-(d-1) i \epsilon \frac{Q^{2} e^{2}}{16 \pi^{2}}\left[A\left(0^{2}\right)+p^{\mu} B_{\mu}\left(p^{2} ; 0^{2}, 0^{2}\right)\right] \\
& =\frac{3-\epsilon}{2} i \epsilon \frac{Q^{2} e^{2}}{16 \pi^{2}} p^{2} B_{0}\left(p^{2} ; 0^{2}, 0^{2}\right) .
\end{aligned}
$$

In the first equality, we take into account the symmetric factor of $1 / 2$. In the second equality, we use $g_{\mu \rho} g_{\nu \sigma} g^{\rho \sigma}\left[-\ell \cdot(\ell+p) g^{\mu \nu}+\ell^{\mu}(\ell+p)^{\nu}\right]=\ell^{2}+\ell \cdot p$.

Figure 1(c) gives

$$
\begin{aligned}
i \mathcal{M}_{(c)}^{\text {loop }}= & i \epsilon\left(i Q e \tilde{\mu}^{\epsilon / 2}\right)^{2} \int \frac{d^{d} \ell}{(2 \pi)^{d}}\left[-\ell \cdot(\ell+p) g^{\mu \nu}+\ell^{\mu}(\ell+p)^{\nu}\right] \frac{-i g_{\mu \rho}}{\ell^{2}} \frac{(-\ell-2 k)^{\rho} i(-\ell-k+q)^{\sigma}}{(\ell+k)^{2}-m^{2}} \frac{-i g_{\nu \sigma}}{(\ell+p)^{2}} \\
= & -4 i \epsilon \frac{Q^{2} e^{2}}{16 \pi^{2}}\left[(k \cdot q) B_{0}\left(q^{2} ; m^{2}, 0^{2}\right)-k^{\mu} q^{\nu} C_{\mu \nu}\left(k^{2}, q^{2}, p^{2} ; 0^{2}, m^{2}, 0^{2}\right)+\left((k \cdot q) k^{\mu}-k^{2} q^{\mu}\right) C_{\mu}\right] \\
= & -i \epsilon \frac{Q^{2} e^{2}}{16 \pi^{2}}\left[A\left(m^{2}\right)+\left(k^{2}+3 k \cdot q+q^{2}+m^{2}\right) B_{0}\left(p^{2} ; 0^{2}, 0^{2}\right)-\left(q^{2}+m^{2}\right) B_{0}\left(q^{2} ; m^{2}, 0^{2}\right)\right. \\
& \left.-\left(k^{2}+m^{2}\right) B_{0}\left(k^{2} ; 0^{2}, m^{2}\right)+\left(4 m^{2} k \cdot q+\left(q^{2}+m^{2}\right)\left(k^{2}+m^{2}\right)\right) C_{0}\right] .
\end{aligned}
$$

In the second equality, we use 


$$
\begin{array}{r}
g_{\mu \rho} g_{\nu \sigma}\left[-\ell \cdot(\ell+p) g^{\mu \nu}+\ell^{\mu}(\ell+p)^{\nu}\right](\ell+2 k)^{\rho}(\ell+k-q)^{\sigma} \\
\quad=4\left[(k \cdot q) \ell^{2}-(\ell \cdot k)(\ell \cdot q)+(k \cdot q)(\ell \cdot k)-k^{2}(\ell \cdot q)\right] .
\end{array}
$$

Here $\ell^{4}$ and $\ell^{3}$ terms vanish because

$$
\left[-\ell \cdot(\ell+p) g^{\mu \nu}+\ell^{\mu}(\ell+p)^{\nu}\right] \ell_{\nu}=(\ell+p)_{\mu}\left[-\ell \cdot(\ell+p) g^{\mu \nu}+\ell^{\mu}(\ell+p)^{\nu}\right]=0 .
$$

We consider the renormalization of a composite operator $|\phi|^{2}$ (mass operator). The renormalized mass operator is written in terms of bare/renormalized fields as follows:

$$
\left|\phi_{0}\right|^{2}=Z_{\phi}|\phi|^{2}=Z_{\phi^{2}}\left[|\phi|^{2}\right]
$$

Here, a square bracket denotes renormalized composite operators. Figure 1(a), with the insertion of $|\phi|^{2}$ instead of $T_{\mu}^{\mu} \supset 2 m^{2}|\phi|^{2}+2 \eta \partial^{2}|\phi|^{2}$, gives the one-loop diagram:

$$
\begin{aligned}
i \mathcal{M}^{\text {loop }}= & i\left(i Q e \tilde{\mu}^{\epsilon / 2}\right)^{2} \int \frac{d^{d} \ell}{(2 \pi)^{d}} \frac{i(\ell-k)^{\nu}}{\ell^{2}-m^{2}} \frac{-i g_{\mu \nu}}{(\ell+k)^{2}} \frac{i(\ell+q+p)^{\mu}}{(\ell+p)^{2}-m^{2}} \\
= & i \frac{Q^{2} e^{2}}{16 \pi^{2}}\left[B_{0}\left(k^{2} ; m^{2}, 0^{2}\right)+B_{0}\left(q^{2} ; 0^{2}, m^{2}\right)-B_{0}\left(p^{2} ; m^{2}, m^{2}\right)\right. \\
& \left.-\left(2 p^{2}-k^{2}-q^{2}-2 m^{2}\right) C_{0}\left(k^{2}, q^{2}, p^{2} ; 0^{2}, m^{2}, 0^{2}\right)\right] .
\end{aligned}
$$

The divergent part is

$$
(i \mathcal{M})_{\mathrm{of} \epsilon}^{\mathrm{pole}}=2 i \frac{Q^{2} e^{2}}{16 \pi^{2}} \frac{1}{\epsilon}
$$

This divergence is canceled by the counterterm,

$$
i \mathcal{M}^{\text {c.t. }}=i\left(Z_{\phi^{2}}^{-1} Z_{\phi}-1\right)
$$

and thus

$$
Z_{\phi^{2}}^{-1} Z_{\phi}-1=-2 \frac{Q^{2} e^{2}}{16 \pi^{2}} \frac{1}{\epsilon}
$$

Since $Z_{\phi}$ at the one-loop level is given in Eq. (B8), one obtains $Z_{\phi^{2}}$ and its anomalous dimension as follows:

$$
Z_{\phi^{2}}-1=6 \frac{Q^{2} e^{2}}{16 \pi^{2}} \frac{1}{\epsilon}, \quad \gamma_{\phi^{2}}=\frac{d \ln Z_{\phi^{2}}}{d \ln \mu}=-6 \frac{Q^{2} e^{2}}{16 \pi^{2}}
$$

\section{Summary of one-loop functions}

One-loop functions are based on Refs. [58,59] (see also Appendix F of Ref. [60]).

The one-point integral is defined as

$$
\tilde{\mu}^{\epsilon} \int \frac{d^{d} \ell}{(2 \pi)^{d}} \frac{1}{\ell^{2}-m^{2}}=\frac{i}{16 \pi^{2}} A\left(m^{2}\right) .
$$

The explicit form is 


$$
A\left(m^{2}\right)=m^{2}\left(\frac{2}{\epsilon}-\ln \left(\frac{m^{2}}{\mu^{2}}\right)+1\right)
$$

Two-point integrals are defined as

$$
\tilde{\mu}^{\epsilon} \int \frac{d^{d} \ell}{(2 \pi)^{d}} \frac{1 ; \ell_{\mu} ; \ell_{\mu} \ell_{\nu}}{\left[\ell^{2}-m_{1}^{2}\right]\left[(\ell+p)^{2}-m_{2}^{2}\right]}=\frac{i}{16 \pi^{2}} B_{0 ; \mu ; \mu \nu}\left(p^{2} ; m_{1}^{2}, m_{2}^{2}\right)
$$

where

$$
B_{\mu}=p_{\mu} B_{1}, \quad B_{\mu \nu}=g_{\mu \nu} B_{22}+p_{\mu} p_{\nu} B_{21}
$$

They can be reduced to $A$ and $B_{0}$ as

$$
\begin{aligned}
B_{1}\left(p^{2} ; m_{1}^{2}, m_{2}^{2}\right) & =\frac{1}{2 p^{2}}\left[A\left(m_{1}^{2}\right)-A\left(m_{2}^{2}\right)-\left(p^{2}+m_{1}^{2}-m_{2}^{2}\right) B_{0}\right], \\
B_{22}\left(p^{2} ; m_{1}^{2}, m_{2}^{2}\right) & =\frac{1}{6}\left[A\left(m_{2}^{2}\right)+2 m_{1}^{2} B_{0}+\left(p^{2}+m_{1}^{2}-m_{2}^{2}\right) B_{1}+m_{1}^{2}+m_{1}^{2}-\frac{p^{2}}{3}\right], \\
B_{21}\left(p^{2} ; m_{1}^{2}, m_{2}^{2}\right) & =\frac{1}{3 p^{2}}\left[A\left(m_{2}^{2}\right)-m_{1}^{2} B_{0}-2\left(p^{2}+m_{1}^{2}-m_{2}^{2}\right) B_{1}-\frac{m_{1}^{2}+m_{1}^{2}}{2}+\frac{p^{2}}{6}\right] .
\end{aligned}
$$

The explicit form with a Feynman parameter integral is

$$
B_{0}=\frac{2}{\epsilon}-\int_{0}^{1} d x \ln \left(\frac{x^{2} p^{2}-x\left(p^{2}+m_{1}^{2}-m_{2}^{2}\right)+m_{1}^{2}-i \epsilon_{\mathrm{ad}}}{\mu^{2}}\right) .
$$

Three-point integrals are defined as

$$
\tilde{\mu}^{\epsilon} \int \frac{d^{d} \ell}{(2 \pi)^{d}} \frac{1 ; \ell_{\mu} ; \ell_{\mu} \ell_{\nu}}{\left[\ell^{2}-m_{1}^{2}\right]\left[(\ell+k)^{2}-m_{2}^{2}\right]\left[(\ell+k+q)^{2}-m_{3}^{2}\right]}=\frac{i}{16 \pi^{2}} C_{0 ; \mu ; \mu \nu}\left(k^{2}, q^{2}, p^{2} ; m_{1}^{2}, m_{2}^{2}, m_{3}^{2}\right)
$$

where $p+q+k=0$ and

$$
C_{\mu}=k_{\mu} C_{11}+q_{\mu} C_{12}, \quad C_{\mu \nu}=g_{\mu \nu} C_{24}+k_{\mu} k_{\nu} C_{21}+q_{\mu} q_{\nu} C_{22}+\left(k_{\mu} q_{\nu}+q_{\mu} k_{\nu}\right) C_{23}
$$

They can be reduced to $A, B_{0}$, and $C_{0}$ as

$$
\begin{aligned}
& C_{11}\left(k^{2}, q^{2}, p^{2} ; m_{1}^{2}, m_{2}^{2}, m_{3}^{2}\right)=\frac{1}{k^{2} q^{2}-(k \cdot q)^{2}}\left[q^{2} R_{1}-(k \cdot q) R_{2}\right] \\
& C_{12}\left(k^{2}, q^{2}, p^{2} ; m_{1}^{2}, m_{2}^{2}, m_{3}^{2}\right)=\frac{1}{k^{2} q^{2}-(k \cdot q)^{2}}\left[-(k \cdot q) R_{1}+k^{2} R_{2}\right] \\
& C_{24}\left(k^{2}, q^{2}, p^{2} ; m_{1}^{2}, m_{2}^{2}, m_{3}^{2}\right)=\frac{1}{4}\left[B_{0}\left(q^{2} ; m_{2}^{2}, m_{3}^{2}\right)+\left(k^{2}+m_{1}^{2}-m_{2}^{2}\right) C_{11}+\left(p^{2}-k^{2}+m_{2}^{2}-m_{3}^{2}\right) C_{12}+2 m_{1}^{2} C_{0}+1\right] \\
& C_{21}\left(k^{2}, q^{2}, p^{2} ; m_{1}^{2}, m_{2}^{2}, m_{3}^{2}\right)=\frac{1}{k^{2} q^{2}-(k \cdot q)^{2}}\left[q^{2} R_{3}-(k \cdot q) R_{5}\right] \\
& C_{23}\left(k^{2}, q^{2}, p^{2} ; m_{1}^{2}, m_{2}^{2}, m_{3}^{2}\right)=\frac{1}{k^{2} q^{2}-(k \cdot q)^{2}}\left[-(k \cdot q) R_{3}+k^{2} R_{5}\right]=\frac{1}{k^{2} q^{2}-(k \cdot q)^{2}}\left[q^{2} R_{4}-(k \cdot q) R_{6}\right], \\
& C_{22}\left(k^{2}, q^{2}, p^{2} ; m_{1}^{2}, m_{2}^{2}, m_{3}^{2}\right)=\frac{1}{k^{2} q^{2}-(k \cdot q)^{2}}\left[-(k \cdot q) R_{4}+k^{2} R_{6}\right],
\end{aligned}
$$

where 


$$
\begin{aligned}
& R_{1}\left(k^{2}, q^{2}, p^{2} ; m_{1}^{2}, m_{2}^{2}, m_{3}^{2}\right)=\frac{1}{2}\left[B_{0}\left(p^{2} ; m_{1}^{2}, m_{3}^{2}\right)-B_{0}\left(q^{2} ; m_{2}^{2}, m_{3}^{2}\right)-\left(k^{2}+m_{1}^{2}-m_{2}^{2}\right) C_{0}\right], \\
& R_{2}\left(k^{2}, q^{2}, p^{2} ; m_{1}^{2}, m_{2}^{2}, m_{3}^{2}\right)=\frac{1}{2}\left[B_{0}\left(k^{2} ; m_{1}^{2}, m_{2}^{2}\right)-B_{0}\left(p^{2} ; m_{1}^{2}, m_{3}^{2}\right)+\left(p^{2}-k^{2}+m_{2}^{2}-m_{3}^{2}\right) C_{0}\right], \\
& R_{3}\left(k^{2}, q^{2}, p^{2} ; m_{1}^{2}, m_{2}^{2}, m_{3}^{2}\right)=-C_{24}-\frac{1}{2}\left[\left(k^{2}+m_{1}^{2}-m_{2}^{2}\right) C_{11}-B_{1}\left(p^{2} ; m_{1}^{2}, m_{3}^{2}\right)-B_{0}\left(q^{2} ; m_{2}^{2}, m_{3}^{2}\right)\right], \\
& R_{4}\left(k^{2}, q^{2}, p^{2} ; m_{1}^{2}, m_{2}^{2}, m_{3}^{2}\right)=-\frac{1}{2}\left[\left(k^{2}+m_{1}^{2}-m_{2}^{2}\right) C_{12}-B_{1}\left(p^{2} ; m_{1}^{2}, m_{3}^{2}\right)+B_{1}\left(q^{2} ; m_{2}^{2}, m_{3}^{2}\right)\right], \\
& R_{5}\left(k^{2}, q^{2}, p^{2} ; m_{1}^{2}, m_{2}^{2}, m_{3}^{2}\right)=-\frac{1}{2}\left[\left(p^{2}-k^{2}+m_{2}^{2}-m_{3}^{2}\right) C_{11}-B_{1}\left(k^{2} ; m_{1}^{2}, m_{2}^{2}\right)+B_{1}\left(p^{2} ; m_{1}^{2}, m_{3}^{2}\right)\right], \\
& R_{6}\left(k^{2}, q^{2}, p^{2} ; m_{1}^{2}, m_{2}^{2}, m_{3}^{2}\right)=-C_{24}-\frac{1}{2}\left[\left(p^{2}-k^{2}+m_{2}^{2}-m_{3}^{2}\right) C_{12}+B_{1}\left(p^{2} ; m_{1}^{2}, m_{3}^{2}\right)\right] .
\end{aligned}
$$

The explicit form with Feynman parameter integrals is ${ }^{2}$

$$
C_{0}=-\int_{0}^{1} d x \int_{0}^{x} d y \frac{1}{x^{2} p^{2}+y^{2} q^{2}-x y\left(p^{2}-k^{2}+q^{2}\right)-x\left(p^{2}+m_{1}^{2}-m_{3}^{2}\right)+y\left(p^{2}-k^{2}+m_{2}^{2}-m_{3}^{2}\right)+m_{1}^{2}-i \epsilon_{\mathrm{ad}}} .
$$

\section{APPENDIX C: TWO-LOOP ORDER IN THE SCALAR QED}

We take a closer look at the two-loop order contributions shown in Fig. 2 with the insertion of $T^{\mu}{ }_{\mu} \supset-(1 / 4) \epsilon F_{\mu \nu}^{2}$. There are two types of diagrams: two-loop diagrams without the insertion of counterterms and one-loop diagrams with the insertion of one-loop counterterms (filled dots in Fig. 2). We consider subdivergences of the former two-loop diagrams. There are two subdivergences in each two-loop diagram, depending on which loop momentum gets large. In the following, we list the corresponding counterterm contributions and check that their sum reproduces that of one-loop diagrams with one-loop counterterms in Fig. 2. We take the massless limit of the scalar $\phi$, $m \rightarrow 0$, since we focus on contributions to the nonminimal coupling.

Figures 3, 4, and 7 show the two-loop diagrams, type (a), (b), (c), and (d) diagrams in Fig. 2, and their subdivergences. We put the two-loop diagrams in the left columns of these figures, while the corresponding counterterm diagrams are in the middle and right columns. The middlecolumn diagrams in Figs. 3, 4 and 7 are with the insertion of counterterms in $T^{\mu}{ }_{\mu}$ (filled-crossed dots). ${ }^{3}$ On the other hand, the right-column diagrams are with the insertion of counterterms for propagators, the $\phi^{*} \phi A$ vertex, and the $\phi^{*} \phi A A$ vertex (unfilled dots). We give the detailed computation of the subdivergences in Appendix C 1.

Among the type (a) and (b) diagrams in Fig. 2, two-loop diagrams are 2(a1), 2(a3), and 2(b1), which are shown in the left column of Fig. 3. For rows of 2(a1) and 2(a3), there

\footnotetext{
${ }^{2}$ The version with $x \rightarrow 1-x$, i.e., $\int_{0}^{1} d x \int_{0}^{1-x} d y \ldots$, may also be familiar to readers.

${ }^{3}$ Precisely speaking, they are not counterterms since they are finite due to $\epsilon$ in $T_{\mu}^{\mu} \supset-(1 / 4) \epsilon F_{\mu \nu}^{2}$.
}

are contributions also from diagrams with internal photons being exchanged. In each row, nonlocal divergences are canceled in light of the BPHZ theorem [6,54,55]. The filled-crossed dots in the middle-column diagrams

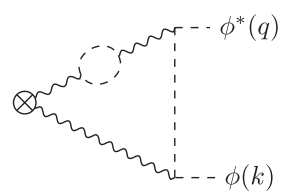

(a1)

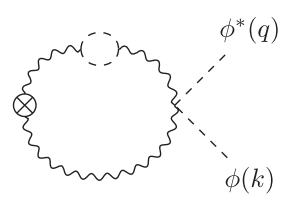

(a3)

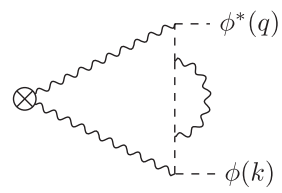

(b1)

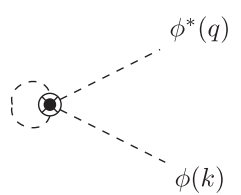

(a1-1)

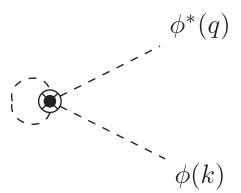

(a3-1)

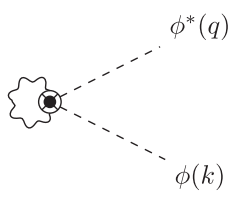

(b1-1)

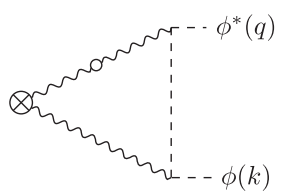

(a2)

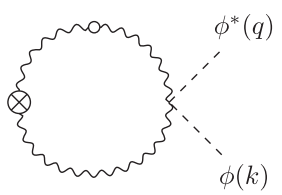

(a4)

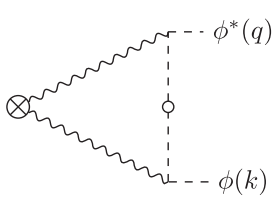

(b2)
FIG. 3. Two-loop diagrams (left column) for scalaron decay $\sigma(p) \rightarrow \phi^{*}(q) \phi(k)$ among the type (a) and (b) diagrams in Fig. 2. For the first two rows, there are contributions also from diagrams with internal photons being exchanged. Crossed dots denote insertion of the energy-momentum tensor, $T^{\mu}{ }_{\mu} \supset-(1 / 4) \epsilon F_{\mu \nu}^{2}$. The corresponding counterterm diagrams are shown in the middle and right columns. The middle-column diagrams are with the insertion of the counterterms in $T_{\mu}^{\mu}$ (filled-crossed dots). The right-column diagrams are with the insertion of the counterterms for propagators (unfilled dots). In each row, nonlocal divergences are canceled. 

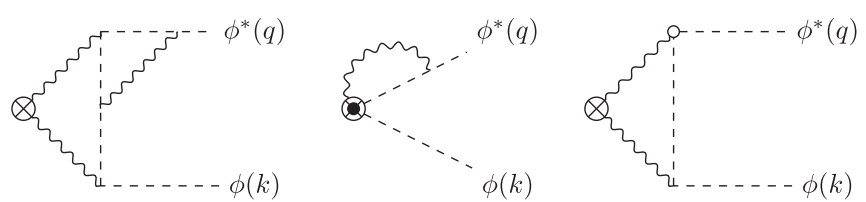

(c1)

(c1-1)

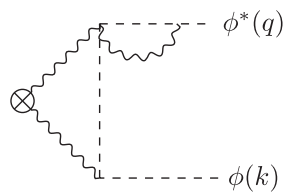

(c2)

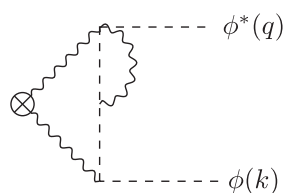

(c3)

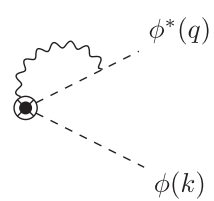

$(\mathrm{c} 2-1)$

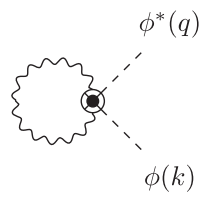

(c3-1) (c2-2)

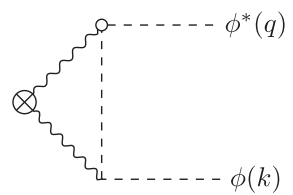

(c3-2)

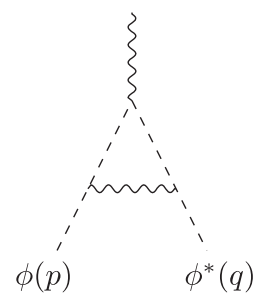

(1)

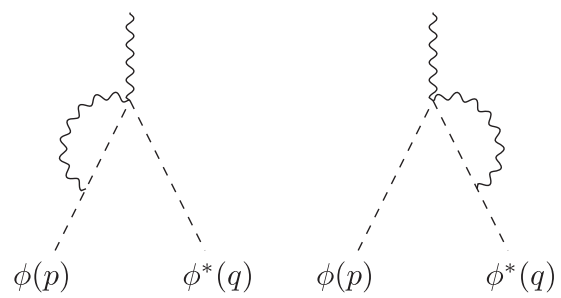

(2)

FIG. 6. One-loop corrections to the $\phi^{*}(q) \phi(p) A_{\mu}$ vertex. Note that $p$ is incoming, while $q$ is outgoing.

FIG. 4. The same as Fig. 3 but for the type (c) diagrams. There are contributions also from diagrams with internal photons being exchanged.

correspond to the insertion of counterterms in $T_{\mu}^{\mu}: 3(\mathrm{a} 1-1)$, $3(\mathrm{a} 3-1) \leftrightarrow|\phi|^{4}$, and 3(b1-1) $\leftrightarrow\left|\phi^{2}\right| A_{\mu}^{2}$. They vanish in the dimensional regularization, since their loop integral is quadratically divergent and contains only massless particles. The right-column diagrams reproduce one-loop diagrams with one-loop counterterms in Fig. 2: 3(a2), $3(\mathrm{a} 4) \leftrightarrow Z_{A}-1$ [see Eq. (B3)], and 3 (b2) $\leftrightarrow Z_{\phi}-1$ [see Eq. (B8)], respectively. In other words, there is no difference between the unfilled dots in Fig. 3 and the filled ones in Fig. 2.

Among the type (c) diagrams in Fig. 2, two-loop diagrams are 2(c1)-2(c3), which are shown in the left column of Fig. 4 [4(c1)-4(c3)]. For each row, there are contributions also from diagrams with internal photons being exchanged. The counterterm [except for 4(c3-1)] (denoted by filled-crossed dots in the middle-column diagrams) cancels with the divergence of the corresponding diagram in Fig. 5: 4(c1-1) ↔5(1), 4(c2-1) $\leftrightarrow 5(2)$ [and $5(3)]$, and 4(c3-1) $\leftrightarrow\left|\phi^{2}\right| A_{\mu}^{2}$. The 4(c1-1) diagram vanishes because Fig. 5(1) is finite and does not have any

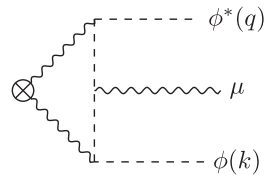

(1)

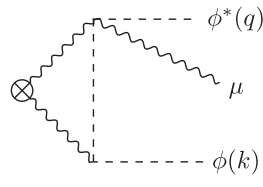

(2)

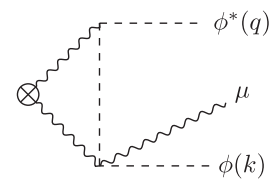

$(3)$

FIG. 5. Type (2) one-loop diagrams with outgoing $\phi^{*}(q) \phi(k) A_{\mu}$ (not necessarily on-shell). Crossed dots denote insertion of $-(1 / 4) \epsilon F_{\mu \nu}^{2}$ with the incoming momentum of $p$.

counterterm. The 4(c2-1) diagram is divergent and may cause a possible issue because there is no corresponding counterterm diagram in Fig. 2. We see that this cancels with other diagrams shortly below. The 4(c3-1) diagram vanishes, because of the quadratically divergent loop integral. The counterterm (unfilled dots in the right-column diagrams) cancels the divergence of each diagram in

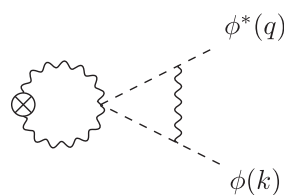

(d1)

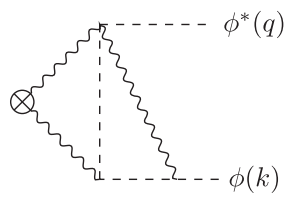

(d2)

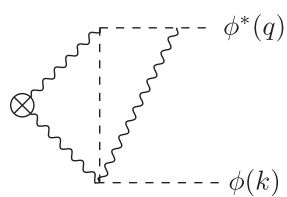

(d3)

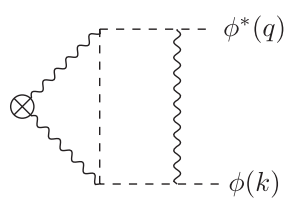

$(\mathrm{d} 4)$

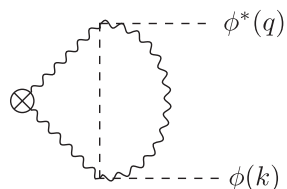

(d5)

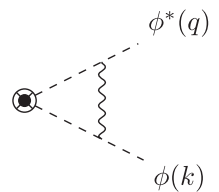

(d1-1)

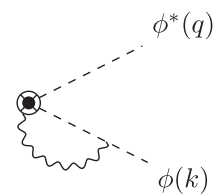

$(\mathrm{d} 2-1)$

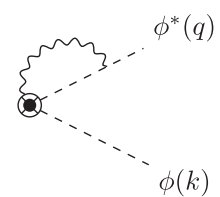

(d3-1)

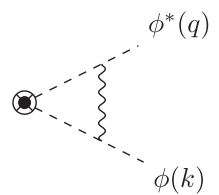

$(\mathrm{d} 4-1)$

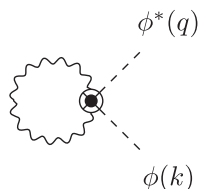

$(\mathrm{d} 5-1)$

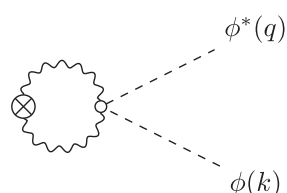

(d1-2)

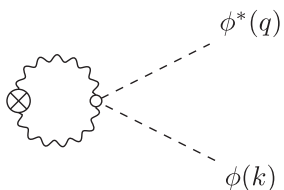

$(\mathrm{d} 2-2)$

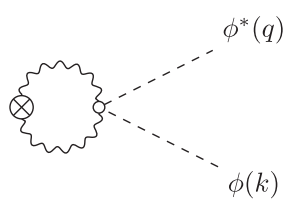

$(\mathrm{d} 3-2)$

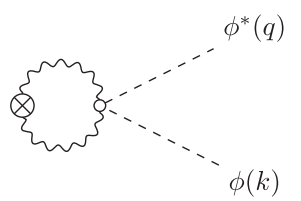

$(d 4-2)$

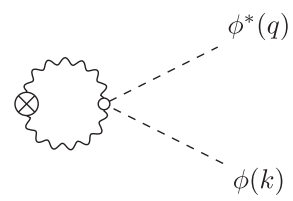

$(\mathrm{d} 5-2)$
FIG. 7. The same as Fig. 3 but for the type (d) diagrams. 
Fig. 6: 4(c1-2) $\leftrightarrow 6(1), 4(\mathrm{c} 2-2) \leftrightarrow 6\left(2^{\prime}\right)$, and 4(c3-2) 6(2). In Appendix $C 1$, we determine the counterterms by evaluating the one-loop corrections to the $\phi^{*}(q) \phi(p) A_{\mu}$ vertex as shown in Fig. 6. Here $p$ is an incoming momentum, while $q$ is outgoing. The results are

$$
\begin{aligned}
& i \Gamma_{3 ; 1 ; \text { c.t. }}^{\mu}(p, q)=\left(i Q e \tilde{\mu}^{\epsilon / 2}\right)(-2)(p+q)^{\mu} \frac{Q^{2} e^{2}}{16 \pi^{2}} \frac{1}{\epsilon}, \\
& i \Gamma_{3 ; 2 ; \text {;.t. }}^{\mu}(p, q)=\left(i Q e \tilde{\mu}^{\epsilon / 2}\right) 6 p^{\mu} \frac{Q^{2} e^{2}}{16 \pi^{2}} \frac{1}{\epsilon}, \\
& i \Gamma_{3 ; 2^{\prime} ; \text {;.t. }}^{\mu}(p, q)=\left(i Q e \tilde{\mu}^{\epsilon / 2}\right) 6 q^{\mu} \frac{Q^{2} e^{2}}{16 \pi^{2}} \frac{1}{\epsilon} .
\end{aligned}
$$

The sum of the counterterms is nothing but the one-loop counterterm of the $\phi^{*} \phi A$ vertex: $\left(i Q e \tilde{\mu}^{\epsilon / 2}\right)\left(Z_{e}-1\right)(p+q)^{\mu}$, where we determine

$$
Z_{e}-1=4 \frac{Q^{2} e^{2}}{16 \pi^{2}} \frac{1}{\epsilon}
$$

from the Ward-Takahashi identity, $Z_{e}=Z_{\phi}$ [see Eq. (B8)]. Thus, the right-column diagrams in Fig. 4 reproduce the one-loop diagram 2(c4) in Fig. 2.

Among the type (d) diagrams in Fig. 2, two-loop diagrams are 2(d1)-2(d5), which are shown in the left column of Fig. 7 [7(d1)-7(d5)]. The counterterm in the middle-column diagrams [except for 7(d5-1)] (denoted by filled-crossed dots) cancels with the divergence of the corresponding diagram in Figs. 1(b) and 1(c) and Fig. 5: $7(\mathrm{~d} 1-1) \leftrightarrow 1(\mathrm{~b}), \quad 7(\mathrm{~d} 2-1) \leftrightarrow 5(2), \quad 7(\mathrm{~d} 3-1) \leftrightarrow 5(3)$, $7(\mathrm{~d} 4-1) \leftrightarrow 1(\mathrm{c})$, and $7(\mathrm{~d} 5-1) \leftrightarrow\left|\phi^{2}\right| A_{\mu}^{2}$. The 7(d1-1)7(d4-1) diagrams are divergent and may cause a possible issue because there is no corresponding counterterm diagram in Fig. 2. We see that this cancels with other diagrams shortly below. The 7(d5-1) diagram vanishes, because of the quadratically divergent loop integral. The counterterm in the right-column diagrams (unfilled dots) cancels the divergence of each diagram in Fig. 8: $7(\mathrm{~d} 1-2) \leftrightarrow 8(1)$, $7(\mathrm{~d} 2-2) \leftrightarrow 8(3), 7(\mathrm{~d} 3-2) \leftrightarrow 8(2), 7(\mathrm{~d} 4-2) \leftrightarrow 8(4)$, and $7(\mathrm{~d} 5-2) \leftrightarrow 8(5)$. In Appendix $\mathrm{C} 1$, we evaluate the $\phi^{*}(q) \phi(p) A_{\mu}(k-p) A_{\nu}$ vertex as shown in Fig. 8. Here $p$ and $k-p$ are incoming momenta, while $q$ is outgoing. For $8(2)-8(5)$, there are contributions with the external photons being exchanged and thus doubled. The resultant counterterms are

$$
\begin{aligned}
& i \Gamma_{4 ; 1 ; \text {;.t. }}^{\mu \nu}=\left(2 i Q^{2} e^{2} \tilde{\mu}^{\epsilon}\right)(-2) g^{\mu \nu} \frac{Q^{2} e^{2}}{16 \pi^{2}} \frac{1}{\epsilon}, \\
& i \Gamma_{4 ; 2 ; \text {;.t. }}^{\mu \nu}=i \Gamma_{4 ; 3 ; \text {;.t. }}^{\mu \nu}=\left(2 i Q^{2} e^{2} \tilde{\mu}^{\epsilon}\right)(-2) g^{\mu \nu} \frac{Q^{2} e^{2}}{16 \pi^{2}} \frac{1}{\epsilon}, \\
& i \Gamma_{4 ; 4 ; \text {;.t. }}^{\mu \nu}=\left(2 i Q^{2} e^{2} \tilde{\mu}^{\epsilon}\right) 2 g^{\mu \nu} \frac{Q^{2} e^{2}}{16 \pi^{2}} \frac{1}{\epsilon}, \\
& i \Gamma_{4 ; 5 ; \text {;.t. }}^{\mu \nu}=\left(2 i Q^{2} e^{2} \tilde{\mu}^{\epsilon}\right) 8 g^{\mu \nu} \frac{Q^{2} e^{2}}{16 \pi^{2}} \frac{1}{\epsilon} .
\end{aligned}
$$

The sum of the counterterms is nothing but the one-loop counterterm of the $\phi^{*} \phi A A$ vertex: $\left(2 i Q^{2} e^{2} \tilde{\mu}^{e}\right)\left(Z_{e^{2}}-1\right) g^{\mu \nu}$, where we determine

$$
Z_{e^{2}}-1=4 \frac{Q^{2} e^{2}}{16 \pi^{2}} \frac{1}{\epsilon}
$$

from the Ward-Takahashi identity, $Z_{e^{2}}=Z_{\phi}$ [see Eq. (B8)]. Thus, the right-column diagrams in Fig. 7 reproduce the one-loop diagram 2(d6) in Fig. 2.

In summary, the right-column diagrams in Figs. 3, 4 and 7 reproduce the one-loop diagrams with the one-loop counterterms in Fig. 2. There are no more diagrams in Fig. 2, and thus the middle-column diagrams in Fig. 3, 4 and 7 should also be zero. On the other hand, the 4(c2-1) (also the one with internal photons being exchanged) and 7(d1-1)-7(d4-1) diagrams are each divergent.

The key is that the counterterms in $T^{\mu}{ }_{\mu}$ form the equation of motion. Each counterterm cancels the divergence of each diagram in Figs. 1(b) and 1(c) and Fig. 5: 4(c2-1) $\leftrightarrow 5(2)$ [and 5(3)], 7(d1-1) ↔ 1(b), 7(d2-1) ↔ 5(2), 7(d3-1) ↔ $5(3)$, and 7(d4-1) $\leftrightarrow 1(\mathrm{c})$. First, Figs. 1(b) and 1(c) [see Eq. (13)] and their counterterms are

$$
\begin{aligned}
& i \mathcal{M}_{2 ;(b) ; \text { c.t. }}=-3 i \frac{Q^{2} e^{2}}{16 \pi^{2}} p^{2}, \\
& i \mathcal{M}_{2 ;(c) ; \text { c.t. }}=6 i \frac{Q^{2} e^{2}}{16 \pi^{2}}(k \cdot q) .
\end{aligned}
$$

By summing them up, we obtain

$$
i \mathcal{M}_{2 ; \text {;.t. }}=-3 i \frac{Q^{2} e^{2}}{16 \pi^{2}}\left(k^{2}+q^{2}\right) .
$$

Note that outgoing $\phi^{*} \phi$ are not necessarily on-shell. Second, we evaluate Fig. 5 in Appendix C 1:

$$
\begin{aligned}
& i \mathcal{M}_{3 ; 2 ; \text { c.t. }}^{\mu}(p, q, k)=\left(i Q e \tilde{\mu}^{\epsilon / 2}\right) 6 \frac{Q^{2} e^{2}}{16 \pi^{2}} k^{\mu}, \\
& i \mathcal{M}_{3 ; 3 ; \text { c.t. }}^{\mu}(p, q, k)=\left(i Q e \tilde{\mu}^{\epsilon / 2}\right)(-6) \frac{Q^{2} e^{2}}{16 \pi^{2}} q^{\mu} .
\end{aligned}
$$

By summing them up, we obtain

$$
i \mathcal{M}_{3 ; \text {;.t. }}^{\mu}(p, q, k)=\left(i Q e \tilde{\mu}^{\epsilon / 2}\right) 6 \frac{Q^{2} e^{2}}{16 \pi^{2}}(k-q)^{\mu}
$$

The total contribution of the counterterms for diagrams with the insertion of $-(1 / 4) \epsilon F_{\mu \nu}^{2}$ are reproduced by the insertion of the equation of motion: 


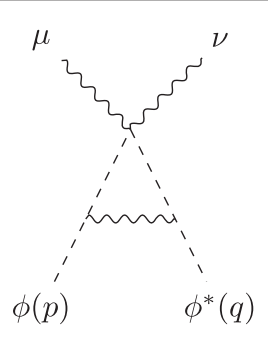

(1)

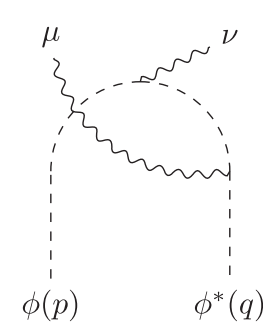

$(2)$

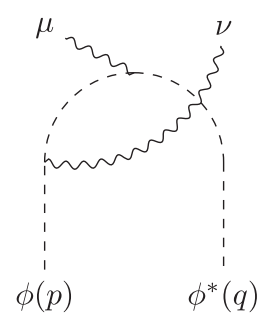

(3)

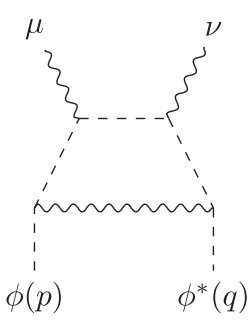

$(4)$

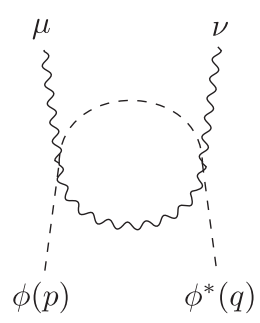

(5)

FIG. 8. One-loop corrections to the $\phi^{*}(q) \phi(p) A_{\mu}(k-p) A_{\nu}$ vertex. Note that $p$ and $k-p$ are incoming, while $q$ is outgoing. For (2)-(5), there are contributions with the external photons being exchanged.

$$
3 \frac{Q^{2} e^{2}}{16 \pi^{2}}\left(\phi^{*} D^{2} \phi+D^{2} \phi^{*} \phi\right)
$$

The insertion of the equation of motion vanishes for the physical amplitude such as scalaron decay $\sigma(p) \rightarrow \phi^{*}(q) \phi(k)$ considered in the main text. [This is why we drop the (e.o.m.) term in Eq. (5).] In conclusion, all the subdivergences, i.e., nonlocal divergences involved in two-loop diagrams of Fig. 2 are canceled by the one-loop diagrams with the one-loop counterterms of Fig. 2 in light of the BPHZ theorem $[6,54,55]$.

\section{One-loop subdiagrams}

Here, we provide the computation of the one-loop subdiagrams, which are illustrated in Figs 5, 6 and 8. We use divergent massless loop integrals summarized in Appendix D 1. First, we evaluate diagrams in Fig. 5 and determined counterterms. Figure 5(1) gives

$$
\begin{aligned}
i \mathcal{M}_{3 ; 1}^{\mu}(p, q, k)= & i \epsilon\left(i Q e \tilde{\mu}^{\epsilon / 2}\right)^{3} \int \frac{d^{d} \ell}{(2 \pi)^{d}} \frac{-i g_{\beta \sigma}}{\ell^{2}}\left[-\ell \cdot(\ell+p) g^{\rho \sigma}+\ell^{\rho}(\ell+p)^{\sigma}\right] \frac{-i g_{\alpha \rho}}{(\ell+p)^{2}}(-\ell-p+2 q)^{\alpha} \\
& \times \frac{i}{(\ell+p-q)^{2}}(-2 \ell-k-p+q)^{\mu} \frac{i}{(\ell+k)^{2}}(-\ell-2 k)^{\beta} \\
= & \left(i Q e \tilde{\mu}^{\epsilon / 2}\right) 4 i \epsilon Q^{2} e^{2} \tilde{\mu}^{\epsilon} \int \frac{d^{d} \ell}{(2 \pi)^{d}} \frac{(k \cdot q) \ell^{2}-(\ell \cdot k)(\ell \cdot q)+(k \cdot q)(\ell \cdot p)-(k \cdot p)(\ell \cdot q)}{\left[\ell^{2}\right]\left[(\ell+p)^{2}\right]\left[(\ell+p-q)^{2}\right]\left[(\ell+k)^{2}\right]} \\
& \times(2 \ell+k+p-q)^{\mu} .
\end{aligned}
$$

Here, $p$ is an incoming momentum, while $q$ and $k$ are outgoing momenta. In the second equality, we use

$$
\begin{aligned}
& g_{\beta \sigma} g_{\alpha \rho}\left[-\ell \cdot(\ell+p) g^{\rho \sigma}+\ell^{\rho}(\ell+p)^{\sigma}\right](\ell+p-2 q)^{\alpha}(\ell+2 k)^{\beta} \\
& \quad=4\left[(k \cdot q) \ell^{2}-(\ell \cdot k)(\ell \cdot q)+(k \cdot q)(\ell \cdot p)-(k \cdot p)(\ell \cdot q)\right] .
\end{aligned}
$$

Thus, $i \mathcal{M}_{3 ; 1}^{\mu}(p, q, k)$ is finite.

Figure 5(2) gives

$$
\begin{aligned}
i \mathcal{M}_{3 ; 2}^{\mu}(p, q, k) & =i \epsilon\left(i Q e \tilde{\mu}^{\epsilon / 2}\right)\left(2 i Q^{2} e^{2} \tilde{\mu}^{\epsilon}\right) \int \frac{d^{d} \ell}{(2 \pi)^{d}} \frac{-i g_{\beta \sigma}}{\ell^{2}}\left[-\ell \cdot(\ell+p) g^{\rho \sigma}+\ell^{\rho}(\ell+p)^{\sigma}\right] \frac{-i g_{\alpha \rho}}{(\ell+p)^{2}} g^{\alpha \mu} \frac{i}{(\ell+k)^{2}}(-\ell-2 k)^{\beta} \\
& =\left(i Q e \tilde{\mu}^{\epsilon / 2}\right) 4 i \epsilon Q^{2} e^{2} \tilde{\mu}^{\epsilon} \int \frac{d^{d} \ell}{(2 \pi)^{d}} \frac{k^{\mu} \ell^{2}-\ell^{\mu}(\ell \cdot k)+k^{\mu}(\ell \cdot p)-\ell^{\mu}(k \cdot p)}{\left[\ell^{2}\right]\left[(\ell+p)^{2}\right]\left[(\ell+k)^{2}\right]}
\end{aligned}
$$

In the second equality, we use

$$
g_{\beta \sigma} g_{\alpha \rho}\left[-\ell \cdot(\ell+p) g^{\rho \sigma}+\ell^{\rho}(\ell+p)^{\sigma}\right] g^{\alpha \mu}(\ell+2 k)^{\beta}=-2\left[k^{\mu} \ell^{2}-\ell^{\mu}(\ell \cdot k)+k^{\mu}(\ell \cdot p)-\ell^{\mu}(k \cdot p)\right] .
$$

We use Eq. (D47) with the replacement of $k_{2} \rightarrow q$ and $\Delta_{x y}=-x y(1-x y) k^{2}+2 x y(1-y) k \cdot p-y(1-y) p^{2}$, 


$$
\left.i \mathcal{M}_{3 ; 2}^{\mu}\right|_{\text {div }}(p, q, k)=\left(i Q e \tilde{\mu}^{\epsilon / 2}\right)(-2) \frac{Q^{2} e^{2}}{16 \pi^{2}} \epsilon e^{\gamma_{E}(\epsilon / 2)} \Gamma(\epsilon / 2)(d-1) k^{\mu}=\left(i Q e \tilde{\mu}^{\epsilon / 2}\right)(-6) \frac{Q^{2} e^{2}}{16 \pi^{2}} k^{\mu}
$$

Figure 5(3) gives

$$
\begin{aligned}
i \mathcal{M}_{3 ; 3}^{\mu}(p, q, k)= & i \epsilon\left(i Q e \tilde{\mu}^{\epsilon / 2}\right)\left(2 i Q^{2} e^{2} \tilde{\mu}^{\epsilon}\right) \int \frac{d^{d} \ell}{(2 \pi)^{d}} \frac{-i g_{\beta \sigma}}{\ell^{2}}\left[-\ell \cdot(\ell+p) g^{\rho \sigma}+\ell^{\rho}(\ell+p)^{\sigma}\right] \frac{-i g_{\alpha \rho}}{(\ell+p)^{2}} \\
& \times(-\ell-p+2 q)^{\alpha} \frac{i}{(\ell+p-q)^{2}} g^{\beta \mu} \\
= & \left(i Q e \tilde{\mu}^{\epsilon / 2}\right)(-4) i \epsilon Q^{2} e^{2} \tilde{\mu}^{\epsilon} \int \frac{d^{d} \ell}{(2 \pi)^{d}} \frac{q^{\mu} \ell^{2}-\ell^{\mu}(\ell \cdot q)+q^{\mu}(\ell \cdot p)-p^{\mu}(\ell \cdot q)}{\left[\ell^{2}\right]\left[(\ell+p)^{2}\right]\left[(\ell+p-q)^{2}\right]} .
\end{aligned}
$$

In the second equality, we use

$$
g_{\beta \sigma} g_{\alpha \rho}\left[-\ell \cdot(\ell+p) g^{\rho \sigma}+\ell^{\rho}(\ell+p)^{\sigma}\right](-\ell-p+2 q)^{\alpha} g^{\beta \mu}=2\left[q^{\mu} \ell^{2}-\ell^{\mu}(\ell \cdot q)+q^{\mu}(\ell \cdot p)-p^{\mu}(\ell \cdot q)\right] .
$$

We use Eq. (D47) with the replacement of $k_{2} \rightarrow q$ and $\Delta_{x y}=-(1-x) y(1-y+x y) p^{2}+2 x(1-x) y^{2} p \cdot q-$ $x y(1-x y) q^{2}$,

$$
\left.i \mathcal{M}_{3 ; 3}^{\mu}\right|_{\text {div }}(p, q, k)=\left(i Q e \tilde{\mu}^{\epsilon / 2}\right) 2 \frac{Q^{2} e^{2}}{16 \pi^{2}} \epsilon e^{\gamma_{E}(\epsilon / 2)} \Gamma(\epsilon / 2)(d-1) q^{\mu}=\left(i Q e \tilde{\mu}^{\epsilon / 2}\right) 6 \frac{Q^{2} e^{2}}{16 \pi^{2}} q^{\mu} .
$$

Second, we compute the one-loop corrections to the $\phi^{*}(q) \phi(p) A_{\mu}$ vertex as shown in Fig. 6. Here $p$ is an incoming momentum, while $q$ is outgoing. Figure 6(1) gives

$$
\begin{aligned}
i \Gamma_{3 ; 1}^{\mu}(p, q) & =\left(i Q e \tilde{\mu}^{\epsilon / 2}\right)^{3} \int \frac{d^{d} \ell}{(2 \pi)^{d}} \frac{-i g_{\rho \sigma}}{\ell^{2}}(\ell+2 p)^{\rho} \frac{i}{(\ell+p)^{2}}(2 \ell+p+q)^{\mu} \frac{i}{(\ell+q)^{2}}(\ell+2 q)^{\sigma} \\
& =\left(i Q e \tilde{\mu}^{\epsilon / 2}\right)(-1) i Q^{2} e^{2} \tilde{\mu}^{\epsilon} \int \frac{d^{d} \ell}{(2 \pi)^{d}} \frac{2 \ell^{\mu} \ell^{2}+4 \ell^{\mu} \ell \cdot(p+q)+(p+q)^{\mu} \ell^{2}+\cdots}{\left[\ell^{2}\right]\left[(\ell+p)^{2}\right]\left[(\ell+q)^{2}\right]} .
\end{aligned}
$$

In the second equality, we keep only the divergent part in the numerator, $(2 \ell+p+q)^{\mu}(\ell+2 p) \cdot(\ell+2 q)$. We use Eq. (D47) with the replacement of $k_{2} \rightarrow q, q_{x y}=(1-y) p+x y q$, and $\Delta_{x y}=-y(1-y) p^{2}+2 x y(1-y) p \cdot q-x y(1-x y) q^{2}$,

$$
\begin{aligned}
\left.i \Gamma_{3 ; 1}^{\mu}\right|_{\operatorname{div}}(p, q) & =\left(i Q e \tilde{\mu}^{\epsilon / 2}\right) \frac{1}{2} \frac{Q^{2} e^{2}}{16 \pi^{2}} e^{\gamma_{E}(\epsilon / 2)} \Gamma(\epsilon / 2) \int_{0}^{1} d x d y y\left[-2(d+2) q_{x y}^{\mu}+4(p+q)^{\mu}+d(p+q)^{\mu}\right]\left(\frac{\mu^{2}}{\Delta_{x y}}\right)^{\epsilon / 2} \\
& =\left(i Q e \tilde{\mu}^{\epsilon / 2}\right) 2(p+q)^{\mu} \frac{Q^{2} e^{2}}{16 \pi^{2}} \frac{1}{\epsilon}
\end{aligned}
$$

In the second equality, we keep only the leading term.

Figures $6(2)$ and $6\left(2^{\prime}\right)$ give

$$
\begin{aligned}
& i \Gamma_{3 ; 2}^{\mu}(p, q)=\left(i Q e \tilde{\mu}^{\epsilon / 2}\right)\left(2 i Q^{2} e^{2} \tilde{\mu}^{\epsilon}\right) \int \frac{d^{d} \ell}{(2 \pi)^{d}} \frac{-i g_{\rho \sigma}}{\ell^{2}} g^{\mu \sigma} \frac{i}{(\ell+p)^{2}}(\ell+2 p)^{\rho}, \\
& i \Gamma_{3 ; 2^{\prime}}^{\mu}(p, q)=\left(i Q e \tilde{\mu}^{\epsilon / 2}\right)\left(2 i Q^{2} e^{2} \tilde{\mu}^{\epsilon}\right) \int \frac{d^{d} \ell}{(2 \pi)^{d}} \frac{-i g_{\rho \sigma}}{\ell^{2}} g^{\mu \sigma} \frac{i}{(\ell+q)^{2}}(\ell+2 q)^{\rho}=i \Gamma_{3 ; 2}^{\mu}(q, p) .
\end{aligned}
$$

We use Eq. (D45) with the replacement of $q_{x}=x p$ and $\Delta_{x}=-x(1-x) p^{2}$ for $i \Gamma_{3 ; 2}^{\mu}(p, q)$ and that of $q_{x}=x q$ and $\Delta_{x}=-x(1-x) q^{2}$ for $i \Gamma_{3 ; 2^{\prime}}^{\mu}(p, q)$, 


$$
\begin{aligned}
\left.i \Gamma_{3 ; 2}^{\mu}\right|_{\mathrm{div}}(p, q) & =\left(i Q e \tilde{\mu}^{\epsilon / 2}\right)(-2) \frac{Q^{2} e^{2}}{16 \pi^{2}} e^{\gamma_{E}(\epsilon / 2)} \Gamma(\epsilon / 2) \int_{0}^{1} d x\left[-q_{x}+2 p^{\mu}\right]\left(\frac{\mu^{2}}{\Delta_{x}}\right)^{\epsilon / 2} \\
& =\left(i Q e \tilde{\mu}^{\epsilon / 2}\right)(-6) p^{\mu} \frac{Q^{2} e^{2}}{16 \pi^{2}} \frac{1}{\epsilon}, \\
\left.i \Gamma_{3 ; 2^{\prime}}^{\mu}\right|_{\mathrm{div}}(p, q) & =\left(i Q e \tilde{\mu}^{\epsilon / 2}\right)(-6) q^{\mu} \frac{Q^{2} e^{2}}{16 \pi^{2}} \frac{1}{\epsilon} .
\end{aligned}
$$

Here, we keep only the leading terms.

Finally, we compute the one-loop corrections to the $\phi^{*}(q) \phi(p) A_{\mu}(k-p) A_{\nu}$ vertex as shown in Fig. 8. Here $p$ and $k-p$ are incoming momenta, while $q$ is outgoing. For $8(2)-8(5)$, there are contributions with the external photons being exchanged. Since we are interested in the divergent part, we take them into account by multiplying 2 in the end of calculations. Figure 8(1) gives

$$
\begin{aligned}
i \Gamma_{4 ; 1}^{\mu \nu} & =\left(2 i Q^{2} e^{2} \tilde{\mu}^{\epsilon}\right)\left(i Q e \tilde{\mu}^{\epsilon / 2}\right)^{2} \int \frac{d^{d} \ell}{(2 \pi)^{d}} \frac{-i g_{\rho \sigma}}{\ell^{2}}(\ell+2 p)^{\rho} \frac{i}{(\ell+p)^{2}} g^{\mu \nu} \frac{i}{(\ell+q)^{2}}(\ell+2 q)^{\sigma} \\
& =\left(2 i Q^{2} e^{2} \tilde{\mu}^{\epsilon}\right)(-1) g^{\mu \nu} i Q^{2} e^{2} \tilde{\mu}^{\epsilon} \int \frac{d^{d} \ell}{(2 \pi)^{d}} \frac{\ell^{2}+\cdots}{\left[\ell^{2}\right]\left[(\ell+p)^{2}\right]\left[(\ell+q)^{2}\right]} .
\end{aligned}
$$

In the second equality, we keep only the divergent part in the numerator, $(\ell+2 p) \cdot(\ell+2 q)$. We use Eq. (D47) with the replacement of $k_{2} \rightarrow q$ and $\Delta_{x y}=-y(1-y) p^{2}+2 x y(1-y) p \cdot q-x y(1-x y) q^{2}$,

$$
\begin{aligned}
\left.i \Gamma_{4 ; 1}^{\mu \nu}\right|_{\mathrm{div}} & =\left(2 i Q^{2} e^{2} \mu^{\epsilon}\right) \frac{d}{4} g^{\mu \nu} \frac{Q^{2} e^{2}}{16 \pi^{2}} e^{\gamma_{E}(\epsilon / 2)} \Gamma(\epsilon / 2) \int_{0}^{1} d x d y 2 y\left(\frac{\mu^{2}}{\Delta_{x y}}\right)^{\epsilon / 2} \\
& =\left(2 i Q^{2} e^{2} \mu^{\epsilon}\right) 2 g^{\mu \nu} \frac{Q^{2} e^{2}}{16 \pi^{2}} \frac{1}{\epsilon} .
\end{aligned}
$$

In the second equality, we keep only the leading term.

Figure 8(2) gives

$$
\begin{aligned}
i \Gamma_{4 ; 2}^{\mu \nu} & =\left(2 i Q^{2} e^{2} \tilde{\mu}^{\epsilon}\right)\left(i Q e \tilde{\mu}^{\epsilon / 2}\right)^{2} \int \frac{d^{d} \ell}{(2 \pi)^{d}} \frac{-i g_{\rho \sigma}}{\ell^{2}} g^{\mu \rho} \frac{i}{(\ell+k)^{2}}(2 \ell+k+q)^{\nu} \frac{i}{(\ell+q)^{2}}(\ell+2 q)^{\sigma} \\
& =\left(2 i Q^{2} e^{2} \tilde{\mu}^{\epsilon}\right)(-1) i Q^{2} e^{2} \tilde{\mu}^{\epsilon} \int \frac{d^{d} \ell}{(2 \pi)^{d}} \frac{2 \ell^{\mu} \ell^{\nu}+\cdots}{\left[\ell^{2}\right]\left[(\ell+k)^{2}\right]\left[(\ell+q)^{2}\right]} .
\end{aligned}
$$

In the second equality, we keep only the divergent part in the numerator, $(\ell+2 q)^{\mu}(2 \ell+k+q)^{\nu}$. We use Eq. (D47), where $k_{2} \rightarrow k, p \rightarrow q$, and $\Delta_{x y}=-x y(1-x y) k^{2}+2 x y(1-y) k \cdot q-y(1-y) q^{2}$,

$$
\left.i \Gamma_{4 ; 2}^{\mu \nu}\right|_{\operatorname{div}}=\left(2 i Q^{2} e^{2} \tilde{\mu}^{\epsilon}\right) \frac{1}{2} \frac{Q^{2} e^{2}}{16 \pi^{2}} e^{\gamma_{E}(\epsilon / 2)} \Gamma(\epsilon / 2) \int_{0}^{1} d x d y 2 y g^{\mu \nu}\left(\frac{\mu^{2}}{\Delta_{x y}}\right)^{\epsilon / 2}=\left(2 i Q^{2} e^{2} \mu^{\epsilon}\right) g^{\mu \nu} \frac{Q^{2} e^{2}}{16 \pi^{2}} \frac{1}{\epsilon} .
$$

In the second equality, we keep only the leading term. This contribution should be doubled to take into account the externalphoton exchange.

Figure 8(3) gives

$$
\begin{aligned}
i \Gamma_{4 ; 3}^{\mu \nu} & =\left(2 i Q^{2} e^{2} \tilde{\mu}^{\epsilon}\right)\left(i Q e \tilde{\mu}^{\epsilon / 2}\right)^{2} \int \frac{d^{d} \ell}{(2 \pi)^{d}} \frac{-i g_{\rho \sigma}}{\ell^{2}}(\ell+2 p)^{\rho} \frac{i}{(\ell+p)^{2}}(2 \ell+k+p)^{\mu} \frac{i}{(\ell+k)^{2}} g^{\nu \sigma} \\
& =\left(2 i Q^{2} e^{2} \mu^{\epsilon}\right)(-1) i Q^{2} e^{2} \tilde{\mu}^{\epsilon} \int \frac{d^{d} \ell}{(2 \pi)^{d}} \frac{2 \ell^{\mu} \ell^{\nu}+\cdots}{\left[\ell^{2}\right]\left[(\ell+k)^{2}\right]\left[(\ell+p)^{2}\right]} .
\end{aligned}
$$

In the second equality, we keep only the divergent part in the numerator, $(2 \ell+k+p)^{\mu}(\ell+2 p)^{\nu}$. We use Eq. (D47) with the replacement of $k_{2} \rightarrow k$ and $\Delta_{x y}=-x y(1-x y) k^{2}+2 x y(1-y) k \cdot p-y(1-y) p^{2}$, 


$$
\left.i \Gamma_{4 ; 3}^{\mu \nu}\right|_{\operatorname{div}}=\left(2 i Q^{2} e^{2} \tilde{\mu}^{\epsilon}\right) \frac{1}{2} \frac{Q^{2} e^{2}}{16 \pi^{2}} e^{\gamma_{E}(\epsilon / 2)} \Gamma(\epsilon / 2) \int_{0}^{1} d x d y 2 y g^{\mu \nu}\left(\frac{\mu^{2}}{\Delta_{x y}}\right)^{\epsilon / 2}=\left(2 i Q^{2} e^{2} \tilde{\mu}^{\epsilon}\right) g^{\mu \nu} \frac{Q^{2} e^{2}}{16 \pi^{2}} \frac{1}{\epsilon} .
$$

In the second equality, we keep only the leading term. This contribution should be doubled to take into account the externalphoton exchange.

Figure 8(4) gives

$$
\begin{aligned}
i \Gamma_{4 ; 4}^{\mu \nu} & =\left(i Q e \tilde{\mu}^{\epsilon / 2}\right)^{4} \int \frac{d^{d} \ell}{(2 \pi)^{d}} \frac{-i g_{\rho \sigma}}{\ell^{2}}(\ell+2 p)^{\rho} \frac{i}{(\ell+p)^{2}}(2 \ell+k+p)^{\mu} \frac{i}{(\ell+k)^{2}}(2 \ell+k+q)^{\nu} \frac{i}{(\ell+q)^{2}}(\ell+2 q)^{\sigma} \\
& =\left(2 i Q^{2} e^{2} \tilde{\mu}^{\epsilon}\right) \frac{1}{2} g_{\rho \sigma} i Q^{2} e^{2} \tilde{\mu}^{\epsilon} \int \frac{d^{d} \ell}{(2 \pi)^{d}} \frac{4 \ell^{\mu} \ell^{\nu} \ell^{\rho} \ell^{\sigma}+\cdots}{\left[\ell^{2}\right]\left[(\ell+k)^{2}\right]\left[(\ell+p)^{2}\right]\left[(\ell+q)^{2}\right]} .
\end{aligned}
$$

In the second equality, we keep only the divergent part in the numerator, $(2 \ell+k+p)^{\mu}(2 \ell+k+q)^{\nu}(\ell+2 p)^{\rho}(\ell+2 q)^{\sigma}$. We use Eq. (D47),

$$
\begin{aligned}
\left.i \Gamma_{4 ; 4}^{\mu \nu}\right|_{\text {div }} & =\left(2 i Q^{2} e^{2} \tilde{\mu}^{\epsilon}\right)(-1) \frac{d+2}{8} \frac{Q^{2} e^{2}}{16 \pi^{2}} e^{\gamma_{E}(\epsilon / 2)} \Gamma(\epsilon / 2) \int_{0}^{1} d x d y d z 4 y z^{2} g^{\mu \nu}\left(\frac{\mu^{2}}{\Delta_{x y}}\right)^{\epsilon / 2} \\
& =\left(2 i Q^{2} e^{2} \mu^{\epsilon}\right)(-1) g^{\mu \nu} \frac{Q^{2} e^{2}}{16 \pi^{2}} \frac{1}{\epsilon} .
\end{aligned}
$$

In the second equality, we keep only the leading term. This contribution should be doubled to take into account the externalphoton exchange.

Figure 8(5) gives

$$
i \Gamma_{4 ; 5}^{\mu \nu}=\left(2 i Q^{2} e^{2} \tilde{\mu}^{\epsilon}\right)^{2} \int \frac{d^{d} \ell}{(2 \pi)^{d}} \frac{-i g_{\rho \sigma}}{\ell^{2}} g^{\mu \rho} \frac{i}{(\ell+k)^{2}} g^{\nu \sigma}=\left(2 i Q^{2} e^{2} \tilde{\mu}^{\epsilon}\right) 2 g^{\mu \nu} i Q^{2} e^{2} \tilde{\mu}^{\epsilon} \int \frac{d^{d} \ell}{(2 \pi)^{d}} \frac{1}{\left[\ell^{2}\right]\left[(\ell+k)^{2}\right]} .
$$

We use Eq. (D45) with the replacement of $p \rightarrow k$ and $\Delta_{x}=-x(1-x) k^{2}$,

$$
\left.i \Gamma_{4 ; 5}^{\mu \nu}\right|_{\mathrm{div}}=\left(2 i Q^{2} e^{2} \tilde{\mu}^{\epsilon}\right)(-2) g^{\mu \nu} \frac{Q^{2} e^{2}}{16 \pi^{2}} e^{\gamma_{E}(\epsilon / 2)} \Gamma(\epsilon / 2) \int_{0}^{1} d x\left(\frac{\mu^{2}}{\Delta_{x}}\right)^{\epsilon / 2}=\left(2 i Q^{2} e^{2} \mu^{\epsilon}\right)(-4) g^{\mu \nu} \frac{Q^{2} e^{2}}{16 \pi^{2}} \frac{1}{\epsilon} .
$$

In the second equality, we keep only the leading term. This contribution should be doubled to take into account the externalphoton exchange.

\section{APPENDIX D: $\beta_{e}$ IN THE QCD}

We consider the QCD whose action is given by

$$
S_{\text {mat }}=\int d^{4} x \sqrt{-g}\left(-\frac{1}{4} g^{\mu \lambda} g^{\nu \kappa} F_{0 \mu \nu}^{a} F_{0 \lambda \kappa}^{a}\right)+S_{\text {fix }},
$$

where we omit the other fields (e.g., quarks) for simplicity. The field strength is

$$
F_{0 \mu \nu}^{a}=\nabla_{\mu} A_{0 \nu}^{a}-\nabla_{\nu} A_{0 \mu}^{a}+e_{0} f^{a b c} A_{0 \mu}^{b} A_{0 \nu}^{c},
$$

with the structure constant $f^{a b c}$. We omit the gauge fixing term $S_{\text {fix }}$ for $T^{\mu \nu}$ as discussed in Ref. [53]. The $d$-dimensional flatspacetime energy-momentum tensor is given by

$$
T_{\mu \nu}=-g^{\lambda \kappa} F_{0 \mu \lambda}^{a} F_{0 \nu \kappa}^{a}+\frac{1}{4} g_{\mu \nu} g^{\lambda \rho} g^{\kappa \sigma} F_{0 \lambda \kappa}^{a} F_{0 \rho \sigma}^{a} .
$$

Taking the trace, one finds 


$$
\begin{aligned}
T_{\mu}^{\mu}= & -\frac{1}{4} \epsilon F_{0 \mu \nu}^{2} \\
= & -\frac{1}{2} \epsilon\left(\partial^{\mu} A^{a \nu}-\partial^{\nu} A^{a \mu}\right) \partial_{\mu} A_{\nu}^{a}-\epsilon \tilde{\mu}^{\epsilon / 2} e f^{a b c} A^{a \mu} A^{b \nu} \partial_{\mu} A_{\nu}^{c}-\frac{1}{4} \epsilon \tilde{\mu}^{\epsilon} e^{2} f^{a b e} f^{c d e} A_{\mu}^{a} A_{\nu}^{b} A^{c \mu} A^{d \nu} \\
& -\frac{1}{2} \epsilon\left(Z_{A}-1\right)\left(\partial^{\mu} A^{a \nu}-\partial^{\nu} A^{a \mu}\right) \partial_{\mu} A_{\nu}^{a}-\epsilon\left(Z_{e}-1\right) \tilde{\mu}^{\epsilon / 2} e f^{a b c} A^{a \mu} A^{b \nu} \partial_{\mu} A_{\nu}^{c} \\
& -\frac{1}{4} \epsilon\left(Z_{e^{2}}-1\right) \tilde{\mu}^{\epsilon} e^{2} f^{a b e} f^{c d e} A_{\mu}^{a} A_{\nu}^{b} A^{c \mu} A^{d \nu} .
\end{aligned}
$$

Here we introduce the multiplicative renormalization,

$$
A_{0 \mu}=Z_{A}^{1 / 2} A_{\mu},
$$

and

$$
\begin{aligned}
Z_{A}^{3 / 2} e_{0} & =Z_{e} \tilde{\mu}^{\epsilon / 2} e, \\
Z_{A}^{2} e_{0}^{2} & \left.=Z_{e^{2}} \tilde{\mu}^{\epsilon} e^{2} \quad \text { (i.e., } Z_{A} Z_{e^{2}}=Z_{e}^{2}\right) .
\end{aligned}
$$

We summarize divergent one-loop integrals in Appendix D 1. We use Feynman-'t Hooft gauge $\left(\xi^{g f}=1\right)$ in the loop calculations.
The scalar and fermion contributions to the wave function renormalization is similar to those in the QED (see Ref. [53]),

$$
Z_{A}-1 \supset\left(-\frac{4}{3} T(F)-\frac{2}{3} T(S)\right) \frac{e^{2}}{16 \pi^{2}} \frac{1}{\epsilon}
$$

except that $Q^{2}$ is replaced by $T(S)$ and $T(F)$ each denoting one-half of the Dynkin index of the representation for the scalar and fermion (Weyl) fields, respectively. There are additional contributions from gauge-boson and ghost loops. The divergent part takes the form of [see Eq. (D45)]

$$
\begin{aligned}
\left.i \Gamma^{(a \mu)(b \nu)}\right|_{\operatorname{div}}(p)= & -e^{2} T(\mathrm{Ad}) \delta^{a b}\left[\frac{i}{16 \pi^{2}} e^{\gamma_{E}(\epsilon / 2)}\left(-\frac{1}{2}\right) \Gamma(-1+\epsilon / 2) \int_{0}^{1} d x h(x) g^{\mu \nu} \Delta_{x}\left(\frac{\mu^{2}}{\Delta_{x}}\right)^{\epsilon / 2}\right. \\
& \left.+\frac{i}{16 \pi^{2}} e^{\gamma_{E}(\epsilon / 2)} \Gamma(\epsilon / 2) \int_{0}^{1} d x\left[f(x) p^{2} g^{\mu \nu}+g(x) p^{\mu} p^{\nu}\right]\left(\frac{\mu^{2}}{\Delta_{x}}\right)^{\epsilon / 2}\right],
\end{aligned}
$$

where $\Delta_{x}=-x(1-x) p^{2}$ and $T(\mathrm{Ad})$ denotes one-half of the Dynkin index of the adjoint representation for the gauge field. We determine $f(x), g(x)$, and $h(x)$ below.

Figure 9 shows contributions from gauge-boson and ghost loops. Figure 9(1) provides

$$
\begin{aligned}
i \Gamma_{1}^{(a \mu)(b \nu)}(p)= & \left(e \mu^{\epsilon / 2}\right)^{2} \frac{1}{2} \int \frac{d^{d} \ell}{(2 \pi)^{d}} \frac{-i g_{\rho \alpha} \delta^{c e}}{\ell^{2}} f^{a c d} V_{e}^{\mu \rho \sigma}(p, \ell,-\ell-p) \frac{-i g_{\sigma \beta} \delta^{d f}}{(\ell+p)^{2}} f^{b e f} V_{e}^{\nu \alpha \beta}(-p,-\ell, \ell+p) \\
= & -e^{2} T(\mathrm{Ad}) \delta^{a b} \mu^{\epsilon} \int \frac{d^{d} \ell}{(2 \pi)^{d}} \frac{1}{\left[\ell^{2}\right]\left[(\ell+p)^{2}\right]}\left[-g^{\mu \nu} \ell^{2}-(2 d-3) \ell^{\mu} \ell^{\nu}\right. \\
& \left.-g^{\mu \nu} \ell \cdot p-\frac{1}{2}(2 d-3) \ell^{\mu} p^{\nu}-\frac{1}{2}(2 d-3) p^{\mu} \ell^{\nu}-\frac{5}{2} g^{\mu \nu} p^{2}+\frac{1}{2}(6-d) p^{\mu} p^{\nu}\right] .
\end{aligned}
$$

In the first equality, we take into account the symmetric factor of $1 / 2 . V_{e}^{\mu \nu \rho}(p, q, k)=g^{\mu \nu}(p-q)^{\rho}+g^{\nu \rho}(q-k)^{\mu}+$ $g^{\rho \mu}(k-p)^{\nu}$ is the vertex factor with $p, q$, and $k$ being

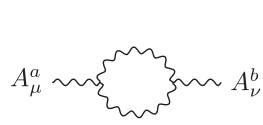

(1)

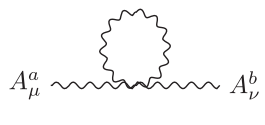

$(2)$

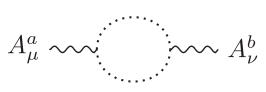

(3)
FIG. 9. One-loop self-energy diagrams for the QCD. The first two diagrams are contributions from the self-interactions of gauge bosons, while the last is a contribution from the ghost interaction. incoming momenta of $A^{a \mu}, A^{b \nu}$, and $A^{c \rho}$, respectively. In the second equality, we use $\delta^{c e} \delta^{d f} f^{a c d} f^{b e f}=T(\mathrm{Ad}) \delta^{a b}$ and

$$
\begin{aligned}
g_{\rho \alpha} g_{\sigma \beta} & V_{e}^{\mu \rho \sigma}(p, \ell,-\ell-p) V_{e}^{\nu \alpha \beta}(-p,-\ell, \ell+p) \\
= & -2 g^{\mu \nu} \ell^{2}-2(2 d-3) \ell^{\mu} \ell^{\nu}-2 g^{\mu \nu} \ell \cdot p \\
& -(2 d-3) \ell^{\mu} p^{\nu}-(2 d-3) p^{\mu} \ell^{\nu}-5 g^{\mu \nu} p^{2} \\
& +(6-d) p^{\mu} p^{\nu} .
\end{aligned}
$$

It contributes to the divergent part as [see Eq. (D45)] 


$$
\begin{aligned}
& h_{1}(x)=-3(d-1), \quad f_{1}(x)=-\frac{5}{2}+x-x^{2}, \\
& g_{1}(x)=\frac{1}{2}(6-d)+(2 d-3) x-(2 d-3) x^{2} .
\end{aligned}
$$

Figure 9(2) provides

$$
\begin{aligned}
& i \Gamma_{2}^{(a \mu)(b \nu)}(p) \\
& \quad=-i e^{2} \tilde{\mu}^{\epsilon} \frac{1}{2} \int \frac{d^{d} \ell}{(2 \pi)^{d}} \frac{-i g_{\rho \sigma} \delta^{c d}}{\ell^{2}} V_{e^{2}}^{(a \mu)(b \nu)(c \rho)(d \sigma)} \\
& \quad=-e^{2} T(\mathrm{Ad}) \delta^{a b} \tilde{\mu}^{\epsilon} \int \frac{d^{d} \ell}{(2 \pi)^{d}} \frac{(d-1)\left(\ell^{2}+2 \ell \cdot p+p^{2}\right) g^{\mu \nu}}{\left[\ell^{2}\right]\left[(\ell+p)^{2}\right]} .
\end{aligned}
$$

In the first equality, we take into account the symmetric factor of $1 / 2 . V_{e^{2}}^{(a \mu)(b \nu)(c \rho)(d \sigma)}=f^{a b e} f^{c d e}\left(g^{\mu \rho} g^{\nu \sigma}-g^{\mu \sigma} g^{\nu \rho}\right)+$ $f^{a c e} f^{b d e}\left(g^{\mu \nu} g^{\rho \sigma}-g^{\mu \sigma} g^{\nu \rho}\right)+f^{a d e} f^{b c e}\left(g^{\mu \nu} g^{\rho \sigma}-g^{\mu \rho} g^{\nu \sigma}\right)$ is the vertex factor for $A^{a \mu}, A^{b \nu}, A^{c \rho}$, and $A^{d \sigma}$. In the second equality, we use $g_{\rho \sigma} \delta^{c d} V_{e^{2}}^{(a \mu)(b \nu)(c \rho)(d \sigma)}=2(d-1) T(\mathrm{Ad}) g^{\mu \nu}$ and multiply $(\ell+p)^{2}$ in both the numerator and the denominator of the integrand. It contributes to the divergent part as [see Eq. (D45)]

$h_{2}(x)=d(d-1)$,

$f_{2}(x)=(d-1)-2(d-1) x+(d-1) x^{2}$,

$g_{2}(x)=0$.

Figure 9(3) provides

$$
\begin{aligned}
i \Gamma_{3}^{(a \mu)(b \nu)}(p)= & \left(-e \mu^{\epsilon / 2}\right)^{2}(-1) \tilde{\mu}^{\epsilon} \int \frac{d^{d} \ell}{(2 \pi)^{d}} \frac{i \delta^{c e}}{\ell^{2}} f^{a c d}(\ell+p)^{\mu} \\
& \times \frac{i \delta^{d f}}{(\ell+p)^{2}} f^{b f e} \ell^{\nu} \\
= & -e^{2} T(\mathrm{Ad}) \delta^{a b} \tilde{\mu}^{\epsilon} \int \frac{d^{d} \ell}{(2 \pi)^{d}} \frac{\ell^{\mu} \ell^{\nu}+p^{\mu} \ell^{\nu}}{\left[\ell^{2}\right]\left[(\ell+p)^{2}\right]} .
\end{aligned}
$$

In the first equality, we take into account the ghost statistics of $(-1)$. In the second equality, we use $\delta^{c e} \delta^{d f} f^{a c d} f^{b f e}=-T(\mathrm{Ad}) \delta^{a b}$. It contributes to the divergent part as [see Eq. (D45)]

$$
h_{3}(x)=1, \quad f_{3}(x)=0, \quad g_{3}(x)=-x+x^{2} .
$$

In summary,

$$
\begin{aligned}
& h(x)=(d-2)^{2}, \quad f(x)=\frac{1}{2}(2 d-7)-(2 d-3) x+(d-2) x^{2}, \\
& g(x)=\frac{1}{2}(6-d)+2(d-2) x-2(d-2) x^{2} .
\end{aligned}
$$

Noting that $(d-2)(-1 / 2) \Gamma(-1+\epsilon / 2)=\Gamma(\epsilon / 2)$ (i.e., no quadratic divergence), we obtain the divergent part of

$$
\begin{aligned}
\left.i \Gamma^{(a \mu)(b \nu)}\right|_{\operatorname{div}}(p) \\
=-i \frac{e^{2}}{16 \pi^{2}} T(\mathrm{Ad}) \delta^{a b} e^{\gamma_{E}(\epsilon / 2)} \Gamma(\epsilon / 2) \\
\quad \times \int_{0}^{1} d x\left[F(x) p^{2} g^{\mu \nu}+G(x) p^{\mu} p^{\nu}\right]\left(\frac{\mu^{2}}{\Delta_{x}}\right)^{\epsilon / 2},
\end{aligned}
$$

where

$$
\begin{aligned}
F(x) & =\left(1-3 x+2 x^{2}\right)(d-4)+\left(\frac{1}{2}-7 x+4 x^{2}\right), \\
G(x) & =\left(-\frac{1}{2}+2 x-2 x^{2}\right)(d-4)+\left(1+4 x-4 x^{2}\right), \\
\int_{0}^{1} d x F(x) & =-\int_{0}^{1} d x G(x)=\frac{1}{6}(d-4)-\frac{5}{3}, \quad \text { (D18) }
\end{aligned}
$$

and thus,

$$
\left(i \Gamma^{(a \mu)(b \nu)}\right)_{\mathrm{of} \epsilon}^{\mathrm{pole}}(p)=i\left(p^{2} g^{\mu \nu}-p^{\mu} p^{\nu}\right) \delta^{a b} \frac{e^{2}}{16 \pi^{2}} \frac{10}{3} T(\mathrm{Ad}) \frac{1}{\epsilon} .
$$

As a result, the wave function renormalization in Feynman-'t Hooft gauge is given by

$Z_{A}-1=\left(\frac{10}{3} T(\mathrm{Ad})-\frac{4}{3} T(F)-\frac{2}{3} T(S)\right) \frac{e^{2}}{16 \pi^{2}} \frac{1}{\epsilon}$.

Meanwhile, the $\beta$ function of the gauge coupling is known to be

$$
\beta_{e}=-\left(\frac{11}{3} T(\mathrm{Ad})-\frac{2}{3} T(F)-\frac{1}{3} T(S)\right) \frac{e^{3}}{16 \pi^{2}} .
$$

Thus, $\epsilon\left(Z_{A}-1\right)=-2 \beta_{e} / e$ does not hold. Precisely speaking, it holds for the scalar and fermion contributions, but not for the gauge boson contribution. We note that $Z_{A}$ is gauge dependent in the $\mathrm{QCD}$, while $\beta_{e}$ is gauge independent. On the other hand, since $T^{\mu}{ }_{\mu}$ is gauge invariant, we expect that the invariant amplitude of scalaron decay into two gauge bosons is proportional to $\beta_{e}$ in the $\mathrm{QCD}$ as in the QED [53]. We directly check it in the following. There, the self-coupling terms of $T^{\mu}{ }_{\mu}$ [second and third terms of Eq. (D4)] become important.

We consider scalaron decay into two gauge bosons, $\sigma(p) \rightarrow A_{\mu}^{a}\left(k_{1}\right) A_{\nu}^{b}\left(k_{2}\right) \quad\left(p\right.$ : incoming momentum; $k_{1}$ and $k_{2}$ : outgoing momenta), at the one-loop level. There are two types of contributions from the insertion of 


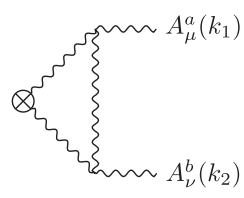

(1)

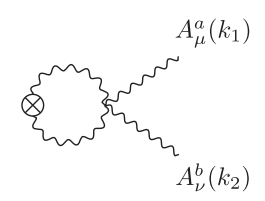

(2)

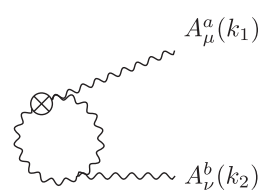

(3)

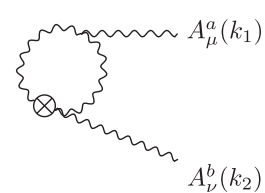

$(3)^{\prime}$

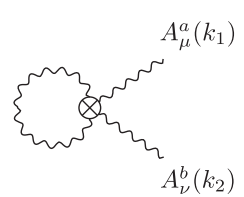

(4)
FIG. 10. Type (2) one-loop diagrams for scalaron decay $\sigma(p) \rightarrow A_{\mu}^{a}\left(k_{1}\right) A_{\nu}^{b}\left(k_{2}\right)$. Crossed dots denote insertion of the energymomentum tensor, (1), (2) -(1/2) $\epsilon\left(\partial^{\mu} A^{a \nu}-\partial^{\nu} A^{a \mu}\right) \partial_{\mu} A_{\nu}^{a}$, (3), (4)

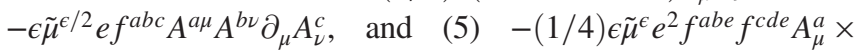
$A_{\nu}^{b} A^{c \mu} A^{d \nu}$.
$T_{\mu}^{\mu} \supset-(1 / 4) \epsilon F_{0 \mu \nu}^{a 2}:(1)$ tree-level diagram from the insertion of $T^{\mu}{ }_{\mu} \supset-(1 / 2) \epsilon\left(Z_{A}-1\right)\left(\partial^{\mu} A^{a \nu}-\partial^{\nu} A^{a \mu}\right) \partial_{\mu} A_{\nu}^{a}$; and (2) one-loop diagrams from the insertion of

$$
\begin{aligned}
T_{\mu}^{\mu}{ }_{\mu} \supset & -(1 / 2) \epsilon\left(\partial^{\mu} A^{a \nu}-\partial^{\nu} A^{a \mu}\right) \partial_{\mu} A_{\nu}^{a} \\
& -\epsilon \tilde{\mu}^{\epsilon / 2} e f^{a b c} A^{a \mu} A^{b \nu} \partial_{\mu} A_{\nu}^{c} \\
& -(1 / 4) \epsilon \tilde{\mu}^{\epsilon} e^{2} f^{a b e} f^{c d e} A_{\mu}^{a} A_{\nu}^{b} A^{c \mu} A^{d \nu} .
\end{aligned}
$$

The type (1) contribution is simply given by

$i \mathcal{M}_{\text {tree }}=i \epsilon\left(Z_{A}-1\right)\left(k_{1} \cdot k_{2} g^{\mu \nu}-k_{2}^{\mu} k_{1}^{\nu}\right) \epsilon_{1 \mu}^{*} \epsilon_{2 \nu}^{*} \delta^{a b}$,

with polarization vectors of $k_{1} \cdot \epsilon_{1}=k_{2} \cdot \epsilon_{2}=0$. The divergent part of the type (2) contributions takes the form of [see Eq. (D47)]

$$
\begin{aligned}
\left.i \mathcal{M}_{\text {loop }}\right|_{\text {div }}= & -\epsilon e^{2} T(\mathrm{Ad}) \delta^{a b} \epsilon_{1 \mu}^{*} \epsilon_{2 \nu}^{*}\left[\frac{i}{16 \pi^{2}} e^{\gamma_{E}(\epsilon / 2)}\left(-\frac{d+2}{4}\right) \Gamma(-1+\epsilon / 2) \int_{0}^{1} d x d y y h(x, y) g^{\mu \nu} \Delta_{x y}\left(\frac{\mu^{2}}{\Delta_{x y}}\right)^{\epsilon / 2}\right. \\
& \left.+\frac{1}{2} \frac{i}{16 \pi^{2}} e^{\gamma_{E}(\epsilon / 2)} \Gamma(\epsilon / 2) \int_{0}^{1} d x d y y\left[f(x, y) k_{1} \cdot k_{2} g^{\mu \nu}+g(x, y) k_{2}^{\mu} k_{1}^{\nu}\right]\left(\frac{\mu^{2}}{\Delta_{x y}}\right)^{\epsilon / 2}\right]
\end{aligned}
$$

where $\Delta_{x y}=-2 y(1-x)(1-y) k_{1} \cdot k_{2}$. Here we use $k_{1} \cdot \epsilon_{1}=k_{2} \cdot \epsilon_{2}=0$ and $k_{1}^{2}=k_{2}^{2}=0$. We determine $f(x, y), g(x, y)$, and $h(x, y)$ below.

Figure 10 shows the type (2) one-loop diagrams. Figures 10(1) and 10(2) are from the insertion of $T_{\mu}^{\mu} \supset-(1 / 2) \epsilon\left(\partial^{\mu} A^{a \nu}-\partial^{\nu} A^{a \mu}\right) \partial_{\mu} A_{\nu}^{a}$. Figure 10(1) gives

$$
\begin{aligned}
i \mathcal{M}_{1}= & i \epsilon\left(e \tilde{\mu}^{\epsilon / 2}\right)^{2} \int \frac{d^{d} \ell}{(2 \pi)^{d}} \frac{-i g_{\beta \sigma} \delta^{h e}}{\ell^{2}}\left[-\ell \cdot(\ell+p) g^{\alpha \beta}+\ell^{\alpha}(\ell+p)^{\beta}\right] \delta^{g h} \frac{-i g_{\alpha \rho} \delta^{g c}}{(\ell+p)^{2}} \\
& \times \epsilon_{1 \mu}^{*} f^{a c d} V_{e}^{\mu \rho \gamma}\left(-k_{1}, \ell+p,-\ell-k_{2}\right) \frac{-i g_{\delta \gamma} \delta^{f d}}{\left(\ell+k_{2}\right)^{2}} \epsilon_{2 \nu}^{*} f^{b e f} V_{e}^{\nu \sigma \delta}\left(-k_{2},-\ell, \ell+k_{2}\right) \\
= & -\epsilon e^{2} T(\mathrm{Ad}) \delta^{a b} \epsilon_{1 \mu}^{*} \epsilon_{2 \nu}^{*} \tilde{\mu}^{\epsilon} \int \frac{d^{d} \ell}{(2 \pi)^{d}} \frac{1}{\left[\ell^{2}\right]\left[\left(\ell+k_{2}\right)^{2}\right]\left[(\ell+p)^{2}\right]}\left[g^{\mu \nu}\left(\ell^{2}\right)^{2}+(4 d-5) \ell^{\mu} \ell^{\nu} \ell^{2}\right. \\
& +g^{\mu \nu} \ell^{2} \ell \cdot\left(3 k_{1}+k_{2}\right)+2 \ell^{\mu} \ell^{\nu} \ell \cdot\left[(2 d-3) k_{1}+2(d-1) k_{2}\right]-\ell^{\mu} k_{1}^{\nu} \ell^{2}+4(d-1) k_{2}^{\mu} \ell^{\nu} \ell^{2} \\
& -6 g^{\mu \nu} \ell^{2} k_{1} \cdot k_{2}+6 g^{\mu \nu}\left(\ell \cdot k_{1}\right) \ell \cdot k_{2}+10 \ell^{\mu} \ell^{\nu} k_{1} \cdot k_{2}-8 \ell^{\mu} k_{1}^{\nu} \ell \cdot k_{2}+4(d-3) k_{2}^{\mu} \ell^{\nu} \ell \cdot k_{1} \\
& \left.+8 k_{2}^{\mu} k_{1}^{\nu} \ell^{2}+\cdots\right] .
\end{aligned}
$$

In the second equality, we use $\delta^{h e} \delta^{g h} \delta^{g c} \delta^{f d} f^{a c d} f^{b e f}=T(\mathrm{Ad}) \delta^{a b}$ and

$$
\begin{aligned}
g_{\beta \sigma} g_{\alpha \rho} & g_{\delta \gamma}\left[-\ell \cdot(\ell+p) g^{\alpha \beta}+\ell^{\alpha}(\ell+p)^{\beta}\right] V_{e}^{\mu \rho \gamma}\left(-k_{1}, \ell+p,-\ell-k_{2}\right) V_{e}^{\nu \sigma \delta}\left(-k_{2},-\ell, \ell+k_{2}\right) \\
= & g^{\mu \nu}\left(\ell^{2}\right)^{2}+(4 d-5) \ell^{\mu} \ell^{\nu} \ell^{2}+g^{\mu \nu} \ell^{2} \ell \cdot\left(3 k_{1}+k_{2}\right)+2 \ell^{\mu} \ell^{\nu} \ell \cdot\left[(2 d-3) k_{1}+2(d-1) k_{2}\right] \\
& +\ell^{\mu}\left[-k_{1}+(2 d-3) k_{2}\right]^{\nu} \ell^{2}+2(d-1)\left(k_{1}+2 k_{2}\right)^{\mu} \ell^{\nu} \ell^{2}-g^{\mu \nu} \ell^{2}\left(6 k_{1} \cdot k_{2}+k_{2}^{2}\right) \\
& +2 g^{\mu \nu}\left(\ell \cdot k_{1}\right) \ell \cdot\left(k_{1}+3 k_{2}\right)+\ell^{\mu} \ell^{\nu}\left(10 k_{1} \cdot k_{2}+4 k_{1}^{2}+5 k_{2}^{2}\right)-2 \ell^{\mu} k_{1}^{\nu} \ell \cdot\left(k_{1}+4 k_{2}\right) \\
& +\ell^{\mu} k_{2}^{\nu} \ell \cdot\left[(2 d-4) k_{1}+(2 d-7) k_{2}\right]+k_{1}^{\mu} \ell^{\nu} \ell \cdot\left[(2 d-7) k_{1}+2(d-1) k_{2}\right] \\
& +4 k_{2}^{\mu} \ell^{\nu} \ell \cdot\left[(d-3) k_{1}+(d-1) k_{2}\right]+\left[k_{1}^{\mu} k_{1}^{\nu}+(d-1) k_{1}^{\mu} k_{2}^{\nu}+8 k_{2}^{\mu} k_{1}^{\nu}+(2 d-1) k_{2}^{\mu} k_{2}^{\nu}\right] \ell^{2}+\cdots
\end{aligned}
$$


Here we omit the linear and zeroth-order terms in $\ell$, which do not give any divergence in the loop integral. In $\mathcal{M}_{1}$, we also omit terms vanishing with $k_{1} \cdot \epsilon_{1}=k_{2} \cdot \epsilon_{2}=0$ and $k_{1}^{2}=k_{2}^{2}=0$ [including $\left(\ell \cdot \epsilon_{1}\right)\left(\ell \cdot k_{1}\right),\left(\ell \cdot k_{1}\right)^{2}$, and so on]. It contributes to the divergent part as [see Eqs. (D47) to (D49)]

$$
\begin{aligned}
& h_{1}(x, y)=5(d-1), \\
& f_{1}(x, y)=2(6 d-1)(1-y)(1-y+x y)-7 d(1-y+x y)-(5 d-2)(1-y)-2(3 d-8), \\
& g_{1}(x, y)=(d+4)(4 d-5)(1-y)(1-y+x y)-(3 d-8)(1-y+x y)-4(d-1)(d+3)(1-y)+4(3 d-5) .
\end{aligned}
$$

Figure 10(2) gives

$$
\begin{aligned}
i \mathcal{M}_{2}= & i \epsilon\left(-i e^{2} \tilde{\mu}^{\epsilon}\right) \frac{1}{2} \int \frac{d^{d} \ell}{(2 \pi)^{d}} \frac{-i g_{\beta \sigma} \delta^{f d}}{\ell^{2}}\left[-\ell \cdot(\ell+p) g^{\alpha \beta}+\ell^{\alpha}(\ell+p)^{\beta}\right] \delta^{e f} \frac{-i g_{\alpha \rho} \delta^{e c}}{(\ell+p)^{2}} \epsilon_{1 \mu}^{*} \epsilon_{2 \nu}^{*} V_{e^{2}}^{(a \mu)(b \nu)(c \rho)(d \sigma)} \\
= & -\epsilon e^{2} T(\mathrm{Ad}) \delta^{a b} \tilde{\mu}^{\epsilon} \int \frac{d^{d} \ell}{(2 \pi)^{d}} \frac{1}{\left[\ell^{2}\right]\left[\left(\ell+k_{2}\right)^{2}\right]\left[(\ell+p)^{2}\right]}\left[-(d-2) g^{\mu \nu}\left(\ell^{2}\right)^{2}-\ell^{\mu} \ell^{\nu} \ell^{2}\right. \\
& -(d-2) g^{\mu \nu} \ell^{2} \ell \cdot\left(k_{1}+3 k_{2}\right)-2 \ell^{\mu} \ell^{\nu} \ell \cdot k_{2}-\frac{1}{2} \ell^{\mu} k_{1}^{\nu} \ell^{2}-\frac{1}{2} k_{2}^{\mu} \ell^{\nu} \ell^{2}-2(d-2) g^{\mu \nu}\left(\ell \cdot k_{1}\right) \ell \cdot k_{2} \\
& \left.-\ell^{\mu} k_{1}^{\nu} \ell \cdot k_{2}-k_{2}^{\mu} \ell^{\nu} \ell \cdot k_{2}+\cdots\right] .
\end{aligned}
$$

In the first equality, we take into account the symmetric factor of $1 / 2$. In the second equality, we multiply $\left(\ell+k_{2}\right)^{2}$ in both the numerator and the denominator of the integrand, and use

$$
\begin{aligned}
& g_{\beta \sigma} g_{\alpha \rho} \delta^{f d} \delta^{e f} \delta^{e c}\left[-\ell \cdot(\ell+p) g^{\alpha \beta}+\ell^{\alpha}(\ell+p)^{\beta}\right] V_{e^{2}}^{(a \mu)(b \nu)(c \rho)(d \sigma)}\left(\ell+k_{2}\right)^{2} \\
&= T(\mathrm{Ad}) \delta^{a b}\left[-2(d-2) g^{\mu \nu} \ell^{2}-2 \ell^{\mu} \ell^{\nu}-2(d-2) g^{\mu \nu} \ell \cdot p-\ell^{\mu} p^{\nu}-p^{\mu} \ell^{\nu}\right]\left(\ell+k_{2}\right)^{2} \\
&= T(\mathrm{Ad}) \delta^{a b}\left[-2(d-2) g^{\mu \nu}\left(\ell^{2}\right)^{2}-2 \ell^{\mu} \ell^{\nu} \ell^{2}-2(d-2) g^{\mu \nu} \ell^{2} \ell \cdot\left(k_{1}+3 k_{2}\right)-4 \ell^{\mu} \ell^{\nu} \ell \cdot k_{2}\right. \\
&-\ell^{\mu}\left(k_{1}+k_{2}\right)^{\nu} \ell^{2}-\left(k_{1}+k_{2}\right)^{\mu} \ell^{\nu} \ell^{2}-4(d-2) g^{\mu \nu}\left[\ell \cdot\left(k_{1}+k_{2}\right)\right] \ell \cdot k_{2}-2(d-2) g^{\mu \nu} \ell^{2} k_{2}^{2} \\
&\left.-\ell^{\mu} \ell^{\nu} k_{2}^{2}-2 \ell^{\mu}\left(k_{1}+k_{2}\right)^{\nu} \ell \cdot k_{2}-2\left(k_{1}+k_{2}\right)^{\mu} \ell^{\nu} \ell \cdot k_{2}+\cdots\right] .
\end{aligned}
$$

Here we omit the linear-order terms in $\ell$, which do not give any divergence in the loop integral. In $\mathcal{M}_{2}$, we also omit terms vanishing with $k_{1} \cdot \epsilon_{1}=k_{2} \cdot \epsilon_{2}=0$ and $k_{1}^{2}=k_{2}^{2}=0$. It contributes to the divergent part as [see Eqs. (D47) to (D49)]

$$
\begin{aligned}
& h_{2}(x, y)=-(d-1)^{2}, \\
& f_{2}(x, y)=-2\left(2 d^{2}-7\right)(1-y)(1-y+x y)+(d-2)(d+2)(1-y+x y)+\left(3 d^{2}-10\right)(1-y)-2(d-2), \\
& g_{2}(x, y)=-(d+4)(1-y)(1-y+x y)+\frac{d+2}{2}(1-y+x y)+\frac{d+6}{2}(1-y)-1 .
\end{aligned}
$$

Figures 10(3) and $10\left(3^{\prime}\right)$ are from the insertion of $T_{\mu}^{\mu} \supset-\epsilon \tilde{\mu}^{\varepsilon / 2} e f^{a b c} A^{a \mu} A^{b \nu} \partial_{\mu} A_{\nu}^{c}$. Figure 10(3) gives

$$
\begin{aligned}
i \mathcal{M}_{3}= & \epsilon e \tilde{\mu}^{\epsilon / 2}\left(e \tilde{\mu}^{\epsilon / 2}\right) \frac{1}{2} \int \frac{d^{d} \ell}{(2 \pi)^{d}} \frac{-i g_{\alpha \rho} \delta^{c e}}{\ell^{2}} \frac{-i g_{\beta \sigma} \delta^{d f}}{\left(\ell+k_{2}\right)^{2}} \epsilon_{1 \mu}^{*} f^{a c d} V_{e}^{\mu \alpha \beta}\left(-k_{1}, \ell,-\ell-k_{2}\right) \epsilon_{2 \nu}^{*} f^{b e f} V_{e}^{\nu \rho \sigma}\left(-k_{2},-\ell, \ell+k_{2}\right) \\
= & -\epsilon e^{2} T(\mathrm{Ad}) \delta^{a b} \tilde{\mu}^{\epsilon} \int \frac{d^{d} \ell}{(2 \pi)^{d}} \frac{1}{\left[\ell^{2}\right]\left[\left(\ell+k_{2}\right)^{2}\right]\left[(\ell+p)^{2}\right]}\left[-g^{\mu \nu}\left(\ell^{2}\right)^{2}-(2 d-3) \ell^{\mu} \ell^{\nu} \ell^{2}\right. \\
& -g^{\mu \nu} \ell^{2} \ell \cdot\left(2 k_{1}+3 k_{2}\right)-2(2 d-3) \ell^{\mu} \ell^{\nu} \ell \cdot\left(k_{1}+k_{2}\right)-\frac{2 d-3}{2} k_{2}^{\mu} \ell^{\nu} \ell^{2}-\frac{1}{2} g^{\mu \nu} \ell^{2} k_{1} \cdot k_{2} \\
& \left.-2 g^{\mu \nu}\left(\ell \cdot k_{1}\right) \ell \cdot k_{2}-2(2 d-3) \ell^{\mu} \ell^{\nu} k_{1} \cdot k_{2}-(2 d-3) k_{2}^{\mu} \ell^{\nu} \ell \cdot k_{1}-\frac{3}{2} k_{2}^{\mu} k_{1}^{\nu} \ell^{2}+\cdots\right] .
\end{aligned}
$$


In the first equality, we take into account the symmetric factor of $1 / 2$. In the second equality, we multiply $\left(\ell+p_{2}\right)^{2}$ in both the numerator and the denominator of the integrand, and we use $\delta^{c e} \delta^{d f} f^{a c d} f^{b e f}=T(\mathrm{Ad}) \delta^{a b}$ and

$$
\begin{aligned}
g_{\alpha \rho} g_{\beta \sigma} & V_{e}^{\mu \alpha \beta}\left(-k_{1}, \ell,-\ell-k_{2}\right) V_{e}^{\nu \rho \sigma}\left(-k_{2},-\ell, \ell+k_{2}\right)(\ell+p)^{2} \\
= & {\left[-2 g^{\mu \nu} \ell^{2}-2(2 d-3) \ell^{\mu} \ell^{\nu}-2 g^{\mu \nu} \ell \cdot k_{2}-(2 d-3) \ell^{\mu} k_{2}^{\nu}-(2 d-3) k_{2}^{\mu} \ell^{\nu}\right.} \\
& \left.+g^{\mu \nu}\left(3 k_{1} \cdot k_{2}-2 k_{2}^{2}\right)-3 k_{2}^{\mu} k_{1}^{\nu}-(d-3) k_{2}^{\mu} k_{2}^{\nu}\right]\left(\ell+k_{1}+k_{2}\right)^{2} \\
= & -2 g^{\mu \nu}\left(\ell^{2}\right)^{2}-2(2 d-3) \ell^{\mu} \ell^{\nu} \ell^{2}-2 g^{\mu \nu} \ell^{2} \ell \cdot\left(2 k_{1}+3 k_{2}\right)-4(2 d-3) \ell^{\mu} \ell^{\nu} \ell \cdot\left(k_{1}+k_{2}\right) \\
& -(2 d-3) \ell^{\mu} k_{2}^{\nu} \ell^{2}-(2 d-3) k_{2}^{\mu} \ell^{\nu} \ell^{2}-g^{\mu \nu} \ell^{2}\left(2 k_{1}^{2}+k_{1} \cdot k_{2}+4 k_{2}^{2}\right)-4 g^{\mu \nu}\left[\ell \cdot\left(k_{1}+k_{2}\right)\right] \ell \cdot k_{2} \\
& -2(2 d-3) \ell^{\mu} \ell^{\nu}\left(k_{1}+k_{2}\right)^{2}-2(2 d-3) \ell^{\mu} k_{2}^{\nu} \ell \cdot\left(k_{1}+k_{2}\right)-2(2 d-3) k_{2}^{\mu} \ell^{\nu} \ell \cdot\left(k_{1}+k_{2}\right) \\
& -3 k_{2}^{\mu} k_{1}^{\nu} \ell^{2}-(d-3) k_{2}^{\mu} k_{2}^{\nu} \ell^{2}+\cdots .
\end{aligned}
$$

Here we omit the linear and zeroth-order terms in $\ell$, which do not give any divergence in the loop integral. In $\mathcal{M}_{3}$, we also omit terms vanishing with $k_{1} \cdot \epsilon_{1}=k_{2} \cdot \epsilon_{2}=0$ and $k_{1}^{2}=k_{2}^{2}=0$. It contributes to the divergent part as [see Eqs. (D47) to (D49)]

$$
\begin{aligned}
& h_{3}(x, y)=-3(d-1) \\
& f_{3}(x, y)=-2(4 d+1)(1-y)(1-y+x y)+2(3 d-1)(1-y+x y)+7 d(1-y)-\frac{9 d-8}{2}, \\
& g_{3}(x, y)=-(d+4)(2 d-3)(1-y)(1-y+x y)+2(2 d-3)(1-y+x y)+\frac{(d+6)(2 d-3)}{2}(1-y)-\frac{7 d-6}{2} .
\end{aligned}
$$

Figure 10(3') gives

$$
\begin{aligned}
i \mathcal{M}_{3^{\prime}}= & \epsilon e \tilde{\mu}^{\epsilon / 2}\left(e \tilde{\mu}^{\epsilon / 2}\right) \frac{1}{2} \int \frac{d^{d} \ell}{(2 \pi)^{d}} \frac{-i g_{\alpha \rho} \delta^{c e}}{(\ell+p)^{2}} \frac{-i g_{\beta \sigma} \delta^{d f}}{\left(\ell+k_{2}\right)^{2}} \epsilon_{1 \mu}^{*} f^{a c d} V_{e}^{\mu \alpha \beta}\left(-k_{1}, \ell+p,-\ell-k_{2}\right) \\
& \times \epsilon_{2 \nu}^{*} f^{b e f} V_{e}^{\nu \rho \sigma}\left(-k_{2},-\ell-p, \ell+k_{2}\right) \\
= & -\epsilon e^{2} T(\mathrm{Ad}) \delta^{a b} \tilde{\mu}^{\epsilon} \int \frac{d^{d} \ell}{(2 \pi)^{d}} \frac{1}{\left[\ell^{2}\right]\left[\left(\ell+k_{2}\right)^{2}\right]\left[(\ell+p)^{2}\right]}\left[-g^{\mu \nu}\left(\ell^{2}\right)^{2}-(2 d-3) \ell^{\mu} \ell^{\nu} \ell^{2}\right. \\
& \left.-g^{\mu \nu} \ell^{2} \ell \cdot\left(k_{1}+2 k_{2}\right)-\frac{2 d-3}{2} \ell^{\mu} k_{1}^{\nu} \ell^{2}-(2 d-3) k_{2}^{\mu} \ell^{\nu} \ell^{2}+\frac{1}{2} g^{\mu \nu} \ell^{2} k_{1} \cdot k_{2}-d \ell^{2} k_{2}^{\mu} k_{1}^{\nu}\right] .
\end{aligned}
$$

In the first equality, we take into account the symmetric factor of $1 / 2$. In the second equality, we multiply $\ell^{2}$ in both the numerator and the denominator of the integrand, and we use $\delta^{c e} \delta^{d f} f^{a c d} f^{b e f}=T(\mathrm{Ad}) \delta^{a b}$ and

$$
\begin{aligned}
g_{\alpha \rho} g_{\beta \sigma} & V_{e}^{\mu \alpha \beta}\left(-k_{1}, \ell+p,-\ell-k_{2}\right) V_{e}^{\nu \rho \sigma}\left(-k_{2},-\ell-p, \ell+k_{2}\right) \\
= & {\left[-2 g^{\mu \nu} \ell^{2}-2(2 d-3) \ell^{\mu} \ell^{\nu}-2 g^{\mu \nu} \ell \cdot\left(k_{1}+2 k_{2}\right)-(2 d-3) \ell^{\mu}\left(k_{1}+2 k_{2}\right)^{\nu}-(2 d-3)\left(k_{1}+2 k_{2}\right)^{\mu} \ell^{\nu}\right.} \\
& \left.-g^{\mu \nu}\left(2 k_{1}^{2}-k_{1} \cdot k_{2}+2 k_{2}^{2}\right)-(d-3) k_{1}^{\mu} k_{1}^{\nu}-(2 d-3) k_{1}^{\mu} k_{2}^{\nu}-2 d k_{2}^{\mu} k_{1}^{\nu}-2(2 d-3) k_{2}^{\mu} k_{2}^{\nu}\right] .
\end{aligned}
$$

In $\mathcal{M}_{3^{\prime}}$, we also omit terms vanishing with $k_{1} \cdot \epsilon_{1}=k_{2} \cdot \epsilon_{2}=0$ and $k_{1}^{2}=k_{2}^{2}=0$. It contributes to the divergent part as [see Eqs. (D47) to (D49)]

$$
\begin{aligned}
h_{3^{\prime}}(x, y)= & -3(d-1), \\
f_{3^{\prime}}(x, y)= & -2(4 d+1)(1-y)(1-y+x y)+(d+2)(1-y+x y)+2(d+2)(1-y)+\frac{d}{2}, \\
g_{3^{\prime}}(x, y)= & -(d+4)(2 d-3)(1-y)(1-y+x y)+\frac{(d+2)(2 d-3)}{2}(1-y+x y) \\
& +(d+2)(2 d-3)(1-y)-d^{2} .
\end{aligned}
$$


Figure 10(4) is from the insertion of ${T^{\mu}}_{\mu} \supset-(1 / 4) \epsilon \tilde{\mu}^{e} e^{2} f^{a b e} f^{c d e} A_{\mu}^{a} A_{\nu}^{b} A^{c \mu} A^{d \nu}$. Figure 10(4) gives

$$
\begin{aligned}
i \mathcal{M}_{4}= & -i \epsilon e^{2} \tilde{\mu}^{\epsilon} \frac{1}{2} \int \frac{d^{d} \ell}{(2 \pi)^{d}} \frac{-i g_{\rho \sigma} \delta^{c d}}{\ell^{2}} V_{e^{2}}^{(a \mu)(b \nu)(c \rho)(d \sigma)} \\
= & -\epsilon e^{2} T(\mathrm{Ad}) \delta^{a b} \tilde{\mu}^{\epsilon} \int \frac{d^{d} \ell}{(2 \pi)^{d}} \frac{1}{\left[\ell^{2}\right]\left[\left(\ell+k_{2}\right)^{2}\right]\left[(\ell+p)^{2}\right]}\left[(d-1) g^{\mu \nu}\left(\ell^{2}\right)^{2}+2(d-1) g^{\mu \nu} \ell^{2} \ell \cdot\left(k_{1}+2 k_{2}\right)\right. \\
& \left.+2(d-1) g^{\mu \nu} \ell^{2} k_{1} \cdot k_{2}+4(d-1) g^{\mu \nu}\left(\ell \cdot k_{1}\right) \ell \cdot k_{2}+\cdots\right] .
\end{aligned}
$$

In the first equality, we take into account the symmetric factor of $1 / 2$. In the second equality, we multiply $\left(\ell+k_{2}\right)^{2}(\ell+$ $p)^{2}$ in both the numerator and the denominator of the integrand, and we use

$$
\begin{aligned}
& g_{\rho \sigma} \delta^{c d} V_{e^{2}}^{(a \mu)(b \nu)(c \rho)(d \sigma)} \\
& \quad=2(d-1) T(\mathrm{Ad}) \delta^{a b} g^{\mu \nu}\left(\ell+k_{2}\right)^{2}\left(\ell+k_{1}+k_{2}\right)^{2} \\
& \quad=2(d-1) T(\mathrm{Ad}) \delta^{a b} g^{\mu \nu}\left[\left(\ell^{2}\right)^{2}+2 \ell^{2} \ell \cdot\left(k_{1}+2 k_{2}\right)+\ell^{2}\left(k_{1}^{2}+2 k_{1} \cdot k_{2}+k_{2}^{2}\right)+4\left[\ell \cdot\left(k_{1}+k_{2}\right)\right] \ell \cdot k_{2}+\cdots\right] .
\end{aligned}
$$

Here we omit the linear and zeroth-order terms, which do not give any divergence in the loop integral. In $\mathcal{M}_{4}$, we also omit terms vanishing with $k_{1} \cdot \epsilon_{1}=k_{2} \cdot \epsilon_{2}=0$ and $k_{1}^{2}=k_{2}^{2}=0$. It contributes to the divergent part as [see Eqs. (D47) to (D49)] $h_{4}(x, y)=d(d-1)$,

$f_{4}(x, y)=4(d-1)(d+2)(1-y)(1-y+x y)-2(d-1)(d+2)(1-y+x y)-4(d-1)(d+2)(1-y)+2(d-1)(d+2)$, $g_{4}(x, y)=0$.

In summary,

$$
\begin{aligned}
& h(x, y)=0 \quad(\text { i.e., no quadratic divergence }) \\
& f(x, y)=-d(d+2)(1-y+x y)-(d-2)(d+2)(1-y)+2\left(d^{2}-5 d+10\right), \\
& g(x, y)=d(d+2)(1-y+x y)-d(d+2)(1-y)-\frac{2 d^{2}-17 d+36}{2} .
\end{aligned}
$$

We obtain the divergent part of

$$
\left.i \mathcal{M}_{\text {loop }}\right|_{\text {div }}=-\epsilon \frac{1}{2} i \frac{e^{2}}{16 \pi^{2}} e^{\gamma_{E}(\epsilon / 2)} \Gamma(\epsilon / 2) T(\operatorname{Ad}) \delta^{a b} \epsilon_{1 \mu}^{*} \epsilon_{2 \nu}^{*} \int_{0}^{1} d x d y y\left[f(x, y) k_{1} \cdot k_{2} g^{\mu \nu}+g(x, y) k_{2}^{\mu} k_{1}^{\nu}\right]\left(\frac{\mu^{2}}{\Delta_{x y}}\right)^{\epsilon / 2},
$$

where

$$
\begin{aligned}
f(x, y) & =(2-x) y(d-4)^{2}-2(6-9 y+5 x y)(d-4)-12(2-3 y+2 x y), \\
g(x, y) & =-(1-x y)(d-4)^{2}+\frac{1+20 x y}{2}(d-4)+24 x y, \\
\int_{0}^{1} d x d y y f(x, y) & =\frac{1}{2}(d-4)^{2}-\frac{5}{3}(d-4)-4, \\
\int_{0}^{1} d x d y y g(x, y) & =-\frac{1}{3}(d-4)^{2}+\frac{23}{12}(d-4)+4,
\end{aligned}
$$

and thus,

$$
i \mathcal{M}_{\text {loop }}=i \frac{e^{2}}{16 \pi^{2}} 4 T(\mathrm{Ad})\left(k_{1} \cdot k_{2} g^{\mu \nu}-k_{2}^{\mu} k_{1}^{\nu}\right) \epsilon_{1 \mu}^{*} \epsilon_{2 \nu}^{*} \delta^{a b} .
$$

Combining the type (1) [see Eq. (D23)] and (2) contributions, we find 


$$
i \mathcal{M}_{\text {tree }}+i \mathcal{M}_{\text {loop }}=i\left(\frac{22}{3} T(\mathrm{Ad})-\frac{4}{3} T(F)-\frac{2}{3} T(S)\right) \frac{e^{2}}{16 \pi^{2}}\left(k_{1} \cdot k_{2} g^{\mu \nu}-k_{2}^{\mu} k_{1}^{\nu}\right) \epsilon_{1 \mu}^{*} \epsilon_{2 \nu}^{*} \delta^{a b},
$$

which is reproduced by the insertion of $\left(\beta_{e} / 2 e\right) F_{\mu \nu}^{a 2}$ [see Eq. (D21)] as expected.

\section{Divergent one-loop integrals}

The two-point integrals are

$$
\begin{aligned}
\left.\tilde{\mu}^{\epsilon} \int \frac{d^{d} \ell}{(2 \pi)^{d}} \frac{1}{\left[\ell^{2}\right]\left[(\ell+p)^{2}\right]}\right|_{\mathrm{div}}= & \frac{i}{16 \pi^{2}} e^{\gamma_{E}(\epsilon / 2)} \Gamma(\epsilon / 2) \int_{0}^{1} d x\left(\frac{\mu^{2}}{\Delta_{x}}\right)^{\epsilon / 2}, \\
\left.\tilde{\mu}^{\epsilon} \int \frac{d^{d} \ell}{(2 \pi)^{d}} \frac{\ell^{\mu}}{\left[\ell^{2}\right]\left[(\ell+p)^{2}\right]}\right|_{\mathrm{div}}= & \frac{i}{16 \pi^{2}} e^{\gamma_{E}(\epsilon / 2)} \Gamma(\epsilon / 2) \int_{0}^{1} d x\left(-q_{x}^{\mu}\right)\left(\frac{\mu^{2}}{\Delta_{x}}\right)^{\epsilon / 2}, \\
\left.\tilde{\mu}^{\epsilon} \int \frac{d^{d} \ell}{(2 \pi)^{d}} \frac{\ell^{\mu} \ell^{\nu}}{\left[\ell^{2}\right]\left[(\ell+p)^{2}\right]}\right|_{\mathrm{div}}= & \frac{i}{16 \pi^{2}} e^{\gamma_{E}(\epsilon / 2)}\left(-\frac{1}{2}\right) \Gamma(-1+\epsilon / 2) \int_{0}^{1} d x g^{\mu \nu} \Delta_{x}\left(\frac{\mu^{2}}{\Delta_{x}}\right)^{\epsilon / 2} \\
& +\frac{i}{16 \pi^{2}} e^{\gamma_{E}(\epsilon / 2)} \Gamma(\epsilon / 2) \int_{0}^{1} d x q_{x}^{\mu} q_{x}^{\nu}\left(\frac{\mu^{2}}{\Delta_{x}}\right)^{\epsilon / 2},
\end{aligned}
$$

where $q_{x}=x p$ and $\Delta_{x}=-x(1-x) p^{2}$.

We use

$$
\frac{1}{A B C}=\int_{0}^{1} d x d y 2 y \frac{1}{[(1-x) y A+x y B+(1-y) C]^{3}} .
$$

The three-point integrals are

$$
\begin{aligned}
\left.\tilde{\mu}^{\epsilon} \int \frac{d^{d} \ell}{(2 \pi)^{d}} \frac{1 ; \ell^{\mu}}{\left[\ell^{2}\right]\left[\left(\ell+k_{2}\right)^{2}\right]\left[(\ell+p)^{2}\right]}\right|_{\text {div }}= & 0, \\
\left.\tilde{\mu}^{\epsilon} \int \frac{d^{d} \ell}{(2 \pi)^{d}} \frac{\ell^{\mu} \ell^{\nu}}{\left[\ell^{2}\right]\left[\left(\ell+k_{2}\right)^{2}\right]\left[(\ell+p)^{2}\right]}\right|_{\text {div }}= & \frac{1}{2} \frac{i}{16 \pi^{2}} e^{\gamma_{E}(\epsilon / 2)} \Gamma(\epsilon / 2) \int_{0}^{1} d x d y y g^{\mu \nu}\left(\frac{\mu^{2}}{\Delta_{x y}}\right)^{\epsilon / 2}, \\
\left.\tilde{\mu}^{\epsilon} \int \frac{d^{d} \ell}{(2 \pi)^{d}} \frac{\ell^{\mu} \ell^{\nu} \ell^{\rho}}{\left[\ell^{2}\right]\left[\left(\ell+k_{2}\right)^{2}\right]\left[(\ell+p)^{2}\right]}\right|_{\text {div }}= & \frac{1}{2} \frac{i}{16 \pi^{2}} e^{\gamma_{E}(\epsilon / 2)} \Gamma(\epsilon / 2) \int_{0}^{1} d x d y y-\left(q_{x y}^{\mu} g^{\nu \rho}+q_{x y}^{\nu} g^{\mu \rho}+q_{x y}^{\rho} g^{\mu \nu}\right)\left(\frac{\mu^{2}}{\Delta_{x y}}\right)^{\epsilon / 2}, \\
\left.\tilde{\mu}^{\epsilon} \int \frac{d^{d} \ell}{(2 \pi)^{d}} \frac{\ell^{2} \ell^{\mu} \ell^{\nu}}{\left[\ell^{2}\right]\left[\left(\ell+k_{2}\right)^{2}\right]\left[(\ell+p)^{2}\right]}\right|_{\mathrm{div}}= & \frac{i}{16 \pi^{2}} e^{\gamma_{E}(\epsilon / 2)}\left(-\frac{d+2}{4}\right) \Gamma(-1+\epsilon / 2) \int_{0}^{1} d x d y y g^{\mu \nu} \Delta_{x y}\left(\frac{\mu^{2}}{\Delta_{x y}}\right)^{\epsilon / 2} \\
& +\frac{1}{2} \frac{i}{16 \pi^{2}} e^{\gamma_{E}(\epsilon / 2)} \Gamma(\epsilon / 2) \int_{0}^{1} d x d y y\left[q_{x y}^{2} g^{\mu \nu}+(d+4) q_{x y}^{\mu} q_{x y}^{\nu}\right]\left(\frac{\mu^{2}}{\Delta_{x y}}\right)^{\epsilon / 2},
\end{aligned}
$$

where $p=k_{1}+k_{2}, q_{x y}=(1-y) k_{1}+(1-y+x y) k_{2}$, and $\Delta_{x y}=-y(1-x)(1-y) p^{2}-x(1-x) y^{2} k_{1}^{2}-x y(1-y) k_{2}^{2}$.

Here are useful expressions when determining $f(x, y), g(x, y)$, and $h(x, y)$. For $k_{1}^{2}=k_{2}^{2}=0$,

$$
q_{x y}^{2}=2(1-y)(1-y+x y) k_{1} \cdot k_{2}, \quad q_{x y} \cdot k_{1}=(1-y+x y) k_{1} \cdot k_{2}, \quad q_{x y} \cdot k_{2}=(1-y) k_{1} \cdot k_{2} .
$$

For the contraction of $\epsilon_{1 \mu} \epsilon_{2 \nu}$ and $k_{1} \cdot \epsilon_{1}=k_{2} \cdot \epsilon_{2}=0$ :

$$
q_{x y}^{\mu} q_{x y}^{\nu}=(1-y)(1-y+x y) k_{2}^{\mu} k_{1}^{\nu}, \quad q_{x y}^{\mu} k_{1}^{\nu}=(1-y+x y) k_{2}^{\mu} k_{1}^{\nu}, \quad k_{2}^{\mu} q_{x y}^{\nu}=(1-y) k_{2}^{\mu} k_{1}^{\nu},
$$

and $k_{1}^{\mu} q_{x y}^{\nu}=q_{x y}^{\mu} k_{2}^{\nu}=0$.

\footnotetext{
${ }^{4}$ The version with $x \rightarrow x /(1-y)$ and $y \rightarrow(1-y)$, i.e., $\int_{0}^{1} d x d y d z \delta(1-x-y-z) \ldots$, may also be familiar to readers.
} 
We use $e^{5}$

$$
\frac{1}{A B C D}=\int_{0}^{1} d x d y d z 6 y z^{2} \frac{1}{[(1-x) y z A+x y z B+(1-z) C+(1-y) z D]^{4}} .
$$

The four-point integral is

$$
\left.\tilde{\mu}^{\epsilon} \int \frac{d^{d} \ell}{(2 \pi)^{d}} \frac{\ell^{2} \ell^{\mu} \ell^{\nu}}{\left[\ell^{2}\right]\left[(\ell+k)^{2}\right]\left[(\ell+p)^{2}\right]\left[(\ell+q)^{2}\right]}\right|_{\text {div }}=\frac{d+2}{4} \frac{i}{16 \pi^{2}} e^{\gamma_{E}(\epsilon / 2)} \Gamma(\epsilon / 2) \int_{0}^{1} d x d y d z y z^{2} g^{\mu \nu}\left(\frac{\mu^{2}}{\Delta_{x y z}}\right)^{\epsilon / 2},
$$

where $\Delta_{x y z}=-x y z(1-x y z) k^{2}-z(1-z) p^{2}-(1-y) z(1+y z-z) q^{2}+2 x y z(1-z) k \cdot p+2 x y(1-y) z^{2} k \cdot q+$ $2(1-y) z(1-z) p \cdot q$.

\footnotetext{
${ }^{5}$ The version with $x \rightarrow x /(1-y-z), y \rightarrow(1-y-z) /(1-z)$, and $z \rightarrow 1-z$, i.e., $\int_{0}^{1} d x d y d z d w \delta(1-x-y-z-w) \ldots$, may also be familiar to readers.
}

[1] C. G. Callan, Jr., S. R. Coleman, and R. Jackiw, A new improved energy-momentum tensor, Ann. Phys. (N.Y.) 59, 42 (1970).

[2] S. R. Coleman and R. Jackiw, Why dilatation generators do not generate dilatations?, Ann. Phys. (N.Y.) 67, 552 (1971).

[3] J. C. Collins, Renormalization of the energy-momentum tensor in $\varphi^{4}$ theory, Phys. Rev. D 14, 1965 (1976).

[4] L. S. Brown and J. C. Collins, Dimensional renormalization of scalar field theory in curved space-time, Ann. Phys. (N.Y.) 130, 215 (1980).

[5] S. J. Hathrell, Trace anomalies and $\lambda \phi^{4}$ theory in curved space, Ann. Phys. (N.Y.) 139, 136 (1982).

[6] W. Zimmermann, Convergence of Bogolyubov's method of renormalization in momentum space, Commun. Math. Phys. 15, 208 (1969); Lect. Notes Phys. 558, 217 (2000).

[7] J. H. Lowenstein, Differential vertex operations in Lagrangian field theory, Commun. Math. Phys. 24, 1 (1971).

[8] J. C. Collins, Normal products in dimensional regularization, Nucl. Phys. B92, 477 (1975).

[9] P. Breitenlohner and D. Maison, Dimensional renormalization and the action principle, Commun. Math. Phys. 52, 11 (1977).

[10] D. Z. Freedman, I. J. Muzinich, and E. J. Weinberg, On the energy-momentum tensor in gauge field theories, Ann. Phys. (N.Y.) 87, 95 (1974).

[11] D. Z. Freedman and E. J. Weinberg, The energy-momentum tensor in scalar and gauge field theories, Ann. Phys. (N.Y.) 87, 354 (1974).

[12] N. K. Nielsen, The energy momentum tensor in a nonabelian quark gluon theory, Nucl. Phys. B120, 212 (1977).

[13] S. L. Adler, J. C. Collins, and A. Duncan, Energymomentum-tensor trace anomaly in spin $1 / 2$ quantum electrodynamics, Phys. Rev. D 15, 1712 (1977).
[14] J. C. Collins, A. Duncan, and S. D. Joglekar, Trace and dilatation anomalies in gauge theories, Phys. Rev. D 16, 438 (1977).

[15] L.S. Brown, Dimensional regularization of composite operators in scalar field theory, Ann. Phys. (N.Y.) 126, 135 (1980).

[16] S. J. Hathrell, Trace anomalies and QED in curved space, Ann. Phys. (N.Y.) 142, 34 (1982).

[17] A. Kamada and T. Kuwahara, Lessons from $T^{\mu}{ }_{\mu}$ on inflation models: Two-scalar theory and Yukawa theory, Phys. Rev. D 101, 096012 (2020).

[18] A. D. Linde, Chaotic inflation, Phys. Lett. 129B, 177 (1983).

[19] G. Hinshaw et al. (WMAP Collaboration), Nine-year Wilkinson microwave anisotropy probe (WMAP) observations: Cosmological parameter results, Astrophys. J. Suppl. 208, 19 (2013).

[20] Y. Akrami et al. (Planck Collaboration), Planck 2018 results. X. Constraints on inflation, Astron. Astrophys. 641, A10 (2020).

[21] A. Linde, M. Noorbala, and A. Westphal, Observational consequences of chaotic inflation with nonminimal coupling to gravity, J. Cosmol. Astropart. Phys. 03 (2011) 013.

[22] L. Boubekeur, E. Giusarma, O. Mena, and H. Ramírez, Do current data prefer a nonminimally coupled inflaton?, Phys. Rev. D 91, 103004 (2015).

[23] M. Shokri, F. Renzi, and A. Melchiorri, Cosmic microwave background constraints on non-minimal couplings in inflationary models with power law potentials, Phys. Dark Universe 24, 100297 (2019).

[24] E. W. Kolb and M. S. Turner, The early Universe, Front. Phys. 69, 1 (1990).

[25] Y. Yoon and Y. Yoon, Asymptotic conformal invariance of $\mathrm{SU}(2)$ and standard models in curved space-time, Int. J. Mod. Phys. A 12, 2903 (1997). 
[26] A. Bilandžić and T. Prokopec, Quantum radiative corrections to slow-roll inflation, Phys. Rev. D 76, 103507 (2007).

[27] A. De Simone, M. P. Hertzberg, and F. Wilczek, Running inflation in the Standard Model, Phys. Lett. B 678, 1 (2009).

[28] F. L. Bezrukov, A. Magnin, and M. Shaposhnikov, Standard Model Higgs boson mass from inflation, Phys. Lett. B 675, 88 (2009).

[29] R. N. Lerner and J. McDonald, Gauge singlet scalar as inflaton and thermal relic dark matter, Phys. Rev. D 80, 123507 (2009).

[30] D. P. George, S. Mooij, and M. Postma, Quantum corrections in Higgs inflation: The real scalar case, J. Cosmol. Astropart. Phys. 02 (2014) 024.

[31] D. P. George, S. Mooij, and M. Postma, Quantum corrections in Higgs inflation: The Standard Model case, J. Cosmol. Astropart. Phys. 04 (2016) 006.

[32] T. Markkanen, T. Tenkanen, V. Vaskonen, and H. Veermäe, Quantum corrections to quartic inflation with a non-minimal coupling: Metric vs. Palatini, J. Cosmol. Astropart. Phys. 03 (2018) 029.

[33] T. Futamase and K.-i. Maeda, Chaotic inflationary scenario of the Universe with a nonminimally coupled "inflaton" field, Phys. Rev. D 39, 399 (1989).

[34] D. Salopek, J. Bond, and J. M. Bardeen, Designing density fluctuation spectra in inflation, Phys. Rev. D 40, 1753 (1989).

[35] J. L. Cervantes-Cota and H. Dehnen, Induced gravity inflation in the standard model of particle physics, Nucl. Phys. B442, 391 (1995).

[36] F. L. Bezrukov and M. Shaposhnikov, The Standard Model Higgs boson as the inflaton, Phys. Lett. B 659, 703 (2008).

[37] C. P. Burgess, H. M. Lee, and M. Trott, Power-counting and the validity of the classical approximation during inflation, J. High Energy Phys. 09 (2009) 103, https://iopscience.iop .org/article/10.1088/1126-6708/2009/09/103.

[38] J. L. F. Barbón and J. R. Espinosa, On the naturalness of Higgs inflation, Phys. Rev. D 79, 081302 (2009).

[39] C. P. Burgess, H. M. Lee, and M. Trott, On Higgs inflation and naturalness, J. High Energy Phys. 07 (2010) 007.

[40] M. P. Hertzberg, On inflation with non-minimal coupling, J. High Energy Phys. 11 (2010) 023.

[41] A. Kehagias, A. M. Dizgah, and A. Riotto, Remarks on the Starobinsky model of inflation and its descendants, Phys. Rev. D 89, 043527 (2014).

[42] Y. Ema, Higgs scalaron mixed inflation, Phys. Lett. B 770, 403 (2017).
[43] D. Gorbunov and A. Tokareva, Scalaron the healer: Removing the strong-coupling in the Higgs- and Higgsdilaton inflations, Phys. Lett. B 788, 37 (2019).

[44] Y. Ema, Dynamical emergence of scalaron in Higgs inflation, J. Cosmol. Astropart. Phys. 09 (2019) 027.

[45] Y. Ema, K. Mukaida, and J. van de Vis, Higgs inflation as nonlinear sigma model and scalaron as its $\sigma$-meson, J. High Energy Phys. 11 (2020) 011.

[46] Y. Ema, K. Mukaida, and J. Van De Vis, Renormalization group equations of Higgs- $\mathrm{R}^{2}$ inflation, J. High Energy Phys. 02 (2021) 109.

[47] K. Fujikawa, Path integral for gauge theories with fermions, Phys. Rev. D 21, 2848 (1980); Erratum, 22, 1499 (1980).

[48] K. Fujikawa, Comment on Chiral and Conformal Anomalies, Phys. Rev. Lett. 44, 1733 (1980).

[49] K. Fujikawa, Energy momentum tensor in quantum field theory, Phys. Rev. D 23, 2262 (1981).

[50] J. F. Ashmore, A method of gauge invariant regularization, Lett. Nuovo Cimento 4, 289 (1972).

[51] G. 't Hooft and M. J. G. Veltman, Regularization and renormalization of gauge fields, Nucl. Phys. B44, 189 (1972).

[52] C. G. Bollini and J. J. Giambiagi, Dimensional renormalization: The number of dimensions as a regularizing parameter, Nuovo Cimento B 12, 20 (1972), https://link.springer .com/article/10.1007/BF02895558.

[53] A. Kamada, On scalaron decay via the trace of energymomentum tensor, J. High Energy Phys. 07 (2019) 172.

[54] N. Bogoliubov and O. Parasiuk, On the multiplication of the causal function in the quantum theory of fields, Acta Math. 97, 227 (1957).

[55] K. Hepp, Proof of the Bogolyubov-Parasiuk theorem on renormalization, Commun. Math. Phys. 2, 301 (1966).

[56] J. Taylor, Ward identities and charge renormalization of the Yang-Mills Field, Nucl. Phys. B33, 436 (1971).

[57] A. Slavnov, Ward identities in gauge theories, Theor. Math. Phys. 10, 99 (1972).

[58] G. 't Hooft and M. J. G. Veltman, Scalar one loop integrals, Nucl. Phys. B153, 365 (1979).

[59] G. Passarino and M. J. G. Veltman, One loop corrections for $\mathrm{e}+\mathrm{e}-$ annihilation into $\mathrm{mu}+\mathrm{mu}-$ in the Weinberg model, Nucl. Phys. B160, 151 (1979).

[60] H. E. Logan, Radiative corrections to the $\mathrm{Z} b$ anti-b vertex and constraints on extended Higgs sectors, Ph.D. thesis, UC, Santa Cruz, 1999, arXiv:hep-ph/9906332. http://wwwlib .umi.com/dissertations/fullcit?p9940272. 